\title{
Article
}

\section{Galaxy And Mass Assembly (GAMA): The mechanisms for quiescent galaxy formation at $z<1$}

Rowlands, K., Wild, V., Bourne, N., Bremer, M., Brough, S., Driver, S. P., Hopkins, A. M., Owers, M. S., Phillipps, S., Pimbblet, K., Sansom, Anne E, Wang, L., Alpaslan, M., Bland-Hawthorn, J., Colless, M., Holwerda, B. W. and Taylor, E. N.

Available at http://clok.uclan.ac.uk/21449/

Rowlands, K., Wild, V., Bourne, N., Bremer, M., Brough, S., Driver, S. P., Hopkins, A. M., Owers, M. S., Phillipps, S. et al (2018) Galaxy And Mass Assembly (GAMA): The mechanisms for quiescent galaxy formation at $z<1$. Monthly Notices of the Royal Astronomical Society, 473 (1). pp. 1168-1185. ISSN 0035-8711

It is advisable to refer to the publisher's version if you intend to cite from the work. http://dx.doi.org/10.1093/mnras/stx1903

For more information about UCLan's research in this area go to http://www.uclan.ac.uk/researchgroups/ and search for <name of research Group>.

For information about Research generally at UCLan please go to http://www.uclan.ac.uk/research/

All outputs in CLoK are protected by Intellectual Property Rights law, including Copyright law. Copyright, IPR and Moral Rights for the works on this site are retained by the individual authors and/or other copyright owners. Terms and conditions for use of this material are defined in the policies page. 


\title{
Galaxy And Mass Assembly (GAMA): The mechanisms for quiescent galaxy formation at $z<1$
}

\author{
K. Rowlands, ${ }^{1,2 \star}$ V. Wild, ${ }^{1}$ N. Bourne,${ }^{3}$ M. Bremer, ${ }^{4}$ S. Brough, ${ }^{5}$ S. P. Driver,${ }^{16}$ \\ A. M. Hopkins ${ }^{5}$ M. S. Owers, ${ }^{5,7}$ S. Phillipps,${ }^{4}$ K. Pimbblet,${ }^{8,9}$ A. E. Sansom, ${ }^{10}$ \\ L. Wang, ${ }^{11,12}$ M. Alpaslan, ${ }^{13}$ J. Bland-Hawthorn, ${ }^{14}$ M. Colless, ${ }^{15}$ B. W. Holwerda ${ }^{16,17}$ \\ and E. N. Taylor ${ }^{18}$
}

Affiliations are listed at the end of the paper

Accepted 2017 July 25. Received 2017 July 20; in original form 2016 November 15

\begin{abstract}
One key problem in astrophysics is understanding how and why galaxies switch off their star formation, building the quiescent population that we observe in the local Universe. From the Galaxy And Mass Assembly and VIsible MultiObject Spectrograph Public Extragalactic Redshift surveys, we use spectroscopic indices to select quiescent and candidate transition galaxies. We identify potentially rapidly transitioning post-starburst (PSB) galaxies and slower transitioning green-valley galaxies. Over the last $8 \mathrm{Gyr}$, the quiescent population has grown more slowly in number density at high masses $\left(\mathrm{M}_{*}>10^{11} \mathrm{M}_{\odot}\right)$ than at intermediate masses $\left(\mathrm{M}_{*}>10^{10.6} \mathrm{M}_{\odot}\right)$. There is evolution in both the PSB and green-valley stellar mass functions, consistent with higher mass galaxies quenching at earlier cosmic times. At intermediate masses $\left(\mathrm{M}_{*}>10^{10.6} \mathrm{M}_{\odot}\right)$, we find a green-valley transition time-scale of $2.6 \mathrm{Gyr}$. Alternatively, at $z \sim 0.7$, the entire growth rate could be explained by fast-quenching PSB galaxies, with a visibility time-scale of $0.5 \mathrm{Gyr}$. At lower redshift, the number density of PSBs is so low that an unphysically short visibility window would be required for them to contribute significantly to the quiescent population growth. The importance of the fast-quenching route may rapidly diminish at $z<1$. However, at high masses $\left(\mathrm{M}_{*}>10^{11} \mathrm{M}_{\odot}\right)$, there is tension between the large number of candidate transition galaxies compared to the slow growth of the quiescent population. This could be resolved if not all high-mass PSB and green-valley galaxies are transitioning from star forming to quiescent, for example if they rejuvenate out of the quiescent population following the accretion of gas and triggering of star formation, or if they fail to completely quench their star formation.
\end{abstract}

Key words: galaxies: evolution-galaxies: interactions - galaxies: luminosity function, mass function-galaxies: starburst-galaxies: star formation-galaxies: statistics.

\section{INTRODUCTION}

The galaxy population displays a colour and morphological bimodality (Strateva et al. 2001; Blanton et al. 2003; Baldry et al. 2004; Bell et al. 2004), which emerged at $z<2$ (e.g. Arnouts et al. 2007; Brammer et al. 2011; Wuyts et al. 2011; Mortlock et al. 2013; Whitaker et al. 2015). Wide-area galaxy surveys have shown that the stellar mass density of the star-forming population has been approximately constant over the last $8 \mathrm{Gyr}(z<1$, e.g. Pozzetti et al. 2010; Ilbert et al. 2013; Moustakas et al. 2013; Muzzin et al. 2013). These recent studies have also charted the growth of

^E-mail: kerowlands.astro@gmail.com the quiescent population over cosmic time, although discrepancies exist at $z<1$ as to how quickly the quiescent population grows. Many studies found that the quiescent population doubled in mass between $0<z<1$ (Bell et al. 2004; Brown et al. 2007; Arnouts et al. 2007). Integrating over galaxies of all masses, Muzzin et al. (2013) found that the quiescent population grows in stellar mass density from $z=1$ to 0.3 , but using the same survey data, Ilbert et al. (2013) found that the number density of quiescent galaxies is flat from $z=1$ to the present day. The growth rate of the quiescent population is likely to be mass dependent; Moustakas et al. (2013) concluded that the number density of quiescent galaxies grows from $z=1$ to now for low mass $\left(\mathbf{M}_{*}<10^{10.6} \mathbf{M}_{\odot}\right)$, but not for higher mass galaxies. For the quiescent population to grow, galaxies must transform from star forming to quiescent, as 
quiescent galaxies are no longer forming stars. Understanding the processes which quench star formation, and the time-scale over which this happens, is one of the major open questions in extragalactic astronomy.

There is much debate about the dominant quenching mechanisms and transition time-scales for galaxies. There are two main quenching channels suggested to halt star formation in galaxies: fast and slow, and while there is no agreement on exactly how fast or how slow these channels are, they are generally linked to different quenching processes (Faber et al. 2007; Fang et al. 2012, 2013; Barro et al. 2013; Yesuf et al. 2014). Star formation in galaxies could quench slowly over many Gyr, where the gas may be stabilized against collapse (e.g. morphological quenching, Martig et al. 2009), or the supply is cut-off and galaxies gradually exhaust their gas through star formation over a time-scale of a few Gyr. For galaxies to stop forming stars more rapidly requires the removal of large amounts of gas. Mergers could be responsible for triggering a chain of events which lead to a more rapid shutdown of star formation in galaxies. Models have shown that the torques induced during a gas-rich major merger might funnel gas towards the galaxy centre, triggering an intense burst of star formation (e.g. Mihos \& Hernquist 1994, 1996; Barnes \& Hernquist 1996), capable of consuming a significant portion of a galaxy's gas supply. The gas is then rapidly depleted, and may additionally be prevented from forming stars via feedback mechanisms (e.g. Benson et al. 2003; Di Matteo, Springel \& Hernquist 2005) from stellar or active galactic nucleus (AGN)-driven winds (e.g. Springel, Di Matteo \& Hernquist 2005; Hopkins et al. 2007; Khalatyan et al. 2008; Kaviraj et al. 2011). Other environment-dependent mechanisms such as ram-pressure stripping (Gunn \& Gott 1972; McCarthy et al. 2008) may also remove the gas reservoir on short-intermediate time-scales.

Observational results on quenching time-scales and mechanisms vary substantially. The dearth of galaxies in the region intermediate between the star-forming and quiescent populations in the optical/ultraviolet (UV) colour-magnitude diagram has often been used to argue that galaxies transition rapidly from star forming to quiescent (e.g. Martin et al. 2007; Kaviraj et al. 2007). Using broadband colours, Schawinski et al. (2014) concluded that disc galaxies quench slowly over many Gyr via gentle, secular processes with little morphological change, whereas spheroidal galaxies undergo faster, more violent quenching which also transforms their morphology. By fitting chemical evolution models to the difference in stellar metallicity between star-forming and quiescent galaxies, Peng, Maiolino \& Cochrane (2015) found that $\mathrm{M}_{*}<10^{11} \mathrm{M}_{\odot}$ galaxies in the local Universe are quenched over a time-scale of $4 \mathrm{Gyr}$, which suggests strangulation is the dominant mechanism, whereby halo gas is removed as a galaxy falls into a group/cluster. Wetzel et al. (2013) found that satellite galaxies continue to form stars for 2$4 \mathrm{Gyr}$ before quenching rapidly in $<0.8 \mathrm{Gyr}$, again leading them to suggest that gas exhaustion (i.e. strangulation) of the gas reservoir is the primary quenching mechanism. Haines et al. (2013) concluded that cluster galaxies are quenched upon infall on time-scales of 0.7-2.0 Gyr, and that slow quenching is suggestive of ram-pressure stripping or starvation mechanisms. The observed decrease in the fraction of star-forming galaxies with increasing environmental density and the independence of star formation rate (SFR) and environment (Wijesinghe et al. 2012; Robotham et al. 2013) suggests that galaxy transformation due to environmental processes must be rapid or have happened long ago (Brough et al. 2013). Cosmological simulations are also starting to provide constraints: Trayford et al. (2016) found in the Evolution and Assembly of GaLaxies and their Environments (EAGLE) simulation that the majority of green-valley galaxies transition over a $<2$ Gyr time-scale. In reality, there is likely to be a diversity in quenching time-scales for galaxies even in the local Universe (Smethurst et al. 2015), see also McGee, Bower \& Balogh (2014) for a compilation of quenching time-scale estimates.

It is clear that the relative importance of the fast and slow quenching channels are not well known, and may change over cosmic time, with stellar mass, and environment (Peng et al. 2010; Wijesinghe et al. 2012; Crossett et al. 2017; Hahn, Tinker \& Wetzel 2016). Such variation may help to explain the diversity of observational results, however, observational methods for identifying quenched and transition galaxies may also be partly responsible. Previous studies have largely relied on broad-band photometric data, with any available spectroscopic data only used to provide a redshift to help with the correction of observed frame colours and environment estimates. Good quality spectroscopic data of galaxy continua contain a wealth of information on the star formation history (SFH) of galaxies, and are arguably better suited to cleanly identifying both fully quenched and transitioning galaxies. In this paper, we fully exploit the spectroscopic data from the Galaxy And Mass Assembly (GAMA) survey and VIMOS Public Extragalactic Redshift Survey (VIPERS) to robustly identify fully quenched and candidate fastand slow-quenching galaxies.

To study galaxies undergoing fast quenching, we need galaxies where we have a good constraint on their recent SFH. Post-starburst (PSB) galaxies, where a galaxy has recently undergone a starburst followed by quenching in the last $1 \mathrm{Gyr}$, are ideal for studying fast quenching. PSB galaxies are sufficiently common at $z \sim 1-2$ that they may contribute significantly to the growth of the red sequence at this important epoch (Wild et al. 2016). It is not well known how much PSBs contribute to the build-up of the quiescent population at $z<1$, due to small number statistics in previous redshift surveys (Blake et al. 2004; Wild et al. 2009; Vergani et al. 2010), and aperture bias in spectroscopic surveys at very low redshifts (Brough et al. 2013; Iglesias-Páramo et al. 2013; Richards et al. 2016). Furthermore, studies of the evolution of the quiescent and green-valley populations have commonly been done using broad-band photometry. In such studies, the sample selection and physical properties can be affected by dust, and there is a larger uncertainty on parameters such as stellar population age, stellar mass and photometric redshift compared to spectroscopic studies. Using spectra allows us to cleanly classify galaxies according to their likely quenching timescales. By identifying large numbers of PSB and green-valley galaxies in large spectroscopic surveys, we can identify which quenching channels are important for building the quiescent population at low redshift.

In this paper, we investigate the mass functions and number density evolution of candidate transition and quenched galaxies at $0<z<1$. This allows us to investigate whether the quiescent galaxy population is growing at $z<1$, and what galaxies are responsible for any growth. We adopt a cosmology with $\Omega_{\mathrm{m}}=0.30, \Omega_{\Lambda}=0.70$ and $H_{o}=70 \mathrm{~km} \mathrm{~s}^{-1} \mathrm{Mpc}^{-1}$.

\section{DATA}

Due to the rarity of PSBs in the local Universe, large-area spectroscopic surveys are required to identify them. Our study necessitates spectra so we can robustly identify quiescent and transition galaxies, a high spectroscopic completeness and a good understanding of the survey selection function. These requirements are met by the GAMA and VIPERS surveys. The GAMA survey allows us to span the range $0.05<z<0.35$, above which only the most 
massive galaxies have adequate signal-to-noise $(\mathrm{S} / \mathrm{N})$ spectra. The VIPERS data allow us to extend our study to higher redshift from $0.5<z<1$. Together these surveys give a total time span of $6.5 \mathrm{Gyr}$ $(0.05<z<1.0)$ to study galaxy evolution.

\subsection{GAMA}

The GAMA survey (Driver et al. 2011; Liske et al. 2015) is a multiwavelength photometric and redshift data base, covering $230 \mathrm{deg}^{2}$ in three equatorial fields at $\sim 9,12$ and $14.5 \mathrm{~h}$ (G09, G12 and G15), and two southern regions (G02 and G23). The GAMA data base provides $r$-band defined matched aperture photometry from the UV-far-infrared (FIR) as described in Hill et al. (2011), Driver et al. (2016) and Wright et al. (2016). In this work, we use the equatorial regions as they are the most spectroscopically complete to $r=19.8 \mathrm{mag}$, which cover $180 \mathrm{deg}^{2}$.

Spectra are obtained for $\sim 250000$ galaxies with a magnitude limit of $r_{\mathrm{AB}}<19.8$ mag mostly using the AAOmega spectrograph (Saunders et al. 2004; Sharp et al. 2006) at the Anglo Australian Telescope. The AAOmega spectra (Hopkins et al. 2013) have a wavelength range of 3750-8850 $\AA$ and a resolution of $R \sim 1100$ at $\sim 4000 \AA$. Additional spectra are included from the Sloan Digital Sky Survey (SDSS, York et al. 2000), which have a wavelength range of 3700-9200 $\AA$ and a resolution of $R \sim 1600$ at $\sim 4000 \AA$. The physical scale covered by the 2 arcsec AAOmega fibres range from $2.0 \mathrm{kpc}$ at $z=0.05$ to $9.9 \mathrm{kpc}$ at $z=0.35$. The 3 arcsec SDSS fibres cover $2.9 \mathrm{kpc}$ at $z=0.05$ and $14.8 \mathrm{kpc}$ at $z=0.35$. We discuss the effects of aperture bias in Section 2.8, but we note that it is minimized by excluding galaxies at $z<0.05$. We do not include GAMA spectra from surveys such as 6dF Galaxy Survey (6dFGS) which are not flux calibrated (Hopkins et al. 2013).

We include all GAMA II main survey galaxies which have science quality redshifts $(n Q>2), 10.0<r_{\text {Petro }}<19.8$ mag and $9.9<\log _{10}\left(\mathrm{M}_{*}\right)<12$ totalling 111477 spectra from $0.05<z<0.35$. These include 97872 GAMA spectra and 13605 SDSS spectra. From this sample, we then excluded 1761 problematic spectra which show, e.g. fibre fringing (identified by eye and through GAMA redshift catalogue flags) and 331 galaxies hosting broad-line AGN from Gordon et al. (2017) and Schneider et al. (2007), which prevents us from robustly measuring spectral features.

In this work, we calculate stellar masses using photometry from the GAMA LambDar Data Release (Driver et al. 2016; Wright et al. 2017). The catalogue comprises deblended matched aperture photometry in 21 bands from the observed frame FUV-FIR, with measurements accounting for differences in pixel scale and point spread function in each band. We utilize the UV FUV and NUV GALEX data, optical ugri magnitudes from SDSS DR6 imaging (Adelman-McCarthy et al. 2008) and near-infrared (NIR) ZYJHK photometry from the Visible and Infrared Telescope for Astronomy (VISTA, Sutherland et al. 2015), as part of the VIsta Kilo-degree INfrared Galaxy survey (VIKING). All photometry has been galactic extinction corrected using the values of $E(B-V)$ derived using the Schlegel, Finkbeiner \& Davis (1998) Galactic extinction maps for a total-to-selective extinction ratio of $R_{\mathrm{V}}=3.1$. For all fluxes, we convolve the catalogue error in quadrature with a calibration error of 10 per cent of the flux to allow for differences in the methods used to measure total photometry and errors in the spectral synthesis models used to fit the underlying stellar populations.

The GAMA and SDSS spectra were taken at a much higher spectral resolution $(R \sim 1100$ and 1600 , respectively) than the VIPERS spectra $(R \sim 210)$. To perform a consistent analysis, we convolve the GAMA/SDSS spectra to the same spectral resolution as the
VIPERS spectra using a Gaussian convolution kernel. During the convolution, we linearly interpolate over bad pixels. In practice, this makes little difference to the galaxy spectra, but does allow us to include more spectra in our analysis which would have important spectral features masked out if we simply propagated the bad pixels in the convolution. We measure the new errors for each convolved spectrum by scaling the unconvolved error array to the standard deviation of the flux in line-free regions of the convolved spectrum at 4200-4300 ̊, to account for covariance between smoothed spectral pixels.

From the GAMA sample, we select galaxies to be at $z>0.05$ so that the higher order Balmer lines ( $\mathrm{H} \delta, \mathrm{H} \epsilon$, etc.) are redshifted into a more sensitive portion of the AAOmega spectrograph, and away from regions at shorter wavelengths where poor flat fielding can affect the spectra. Additionally, at $z>0.05$, the fibre samples a substantial fraction of the galaxy light (10-30 per cent of the Petrosian radius) and so minimizes aperture effects (see Section 2.8 and Kewley, Jansen \& Geller 2005). The upper redshift limit is set to $z=0.35$ as above this the mass completeness limit exceeds $\mathrm{M}_{*}>10^{11}$ in some spectral classes, leaving us with few galaxies to study. Note that the $3750-4150 \AA$ region (used for spectral classification, see Section 2.3) is required to always be in the observed spectral range.

\subsection{VIPERS}

The VIPERS Public Data Release $1^{1}$ (PDR1) provides $61221 \mathrm{spec}-$ tra for galaxies with $17.5<i_{\mathrm{AB}}<22.5 \mathrm{mag}$. The PDR1 covers $10.315 \mathrm{deg}^{2}$ (after accounting for the photometric and spectroscopic masks) in the Canada-France-Hawaii Telescope Legacy Survey Wide (CFHTLS-Wide) W1 and W4 fields. A colour selection using $(g-r)$ and $(r-i)$ cuts was used to primarily select galaxies in the range $0.5<z<1.3$. Spectra were observed using the VIsible MultiObject Spectrograph (VIMOS) spectrograph on the Very Large Telescope (VLT) with the LR-grism, yielding a spectral resolution of $R \sim 210$ (at $\sim 6000 \AA$ ) with wavelength coverage from 5500$9500 \AA$ A. Further details of the survey data are given in Guzzo et al. (2014) and Garilli et al. (2014). The VIPERS survey used a slit with 1 arcsec width, but with considerably longer length. The majority of each galaxy should be in each slit and any aperture bias between the GAMA and VIPERS samples should be negligible, except at the lowest redshifts in the GAMA sample.

To calculate stellar masses (see Section 2.4), we use total broadband photometry in the $F U V, N U V, u^{\star}, g^{\prime}, r^{\prime}, i^{\prime}, z^{\prime}$ and $K_{s}$ bands measured using SExTRACTOR MAG_AUTO as described in Moutard et al. (2016) . All photometry has been galactic extinction corrected using $E(B-V)$ values of 0.025 in the W1 field and 0.05 in the W4 field (Fritz et al. 2014), derived using the Schlegel et al. (1998) Galactic extinction maps for a total-to-selective extinction ratio of $R_{\mathrm{V}}=3.1$. Wide-field InfraRed Camera $K_{\mathrm{S}}$ band data are available for 91.5 per cent galaxies. We checked that the lack of NIR data for some galaxies does not significantly affect our stellar mass estimates. For all fluxes, we convolve the catalogue error in quadrature with a calibration error of 10 per cent of the flux to allow for differences in the methods used to measure total photometry and errors in the spectral synthesis models used to fit the underlying stellar populations.

We use galaxies with $0.5<z<1.0,9.9<\log _{10}\left(\mathrm{M}_{*}\right)<12$, and which have secure spectroscopic redshifts with flags $2.0 \leq z_{\text {flg }} \leq 9.5$ (corresponding to a 95 per cent confidence limit on the redshift) and

\footnotetext{
${ }^{1} \mathrm{http}: / /$ vipers.inaf.it/
} 

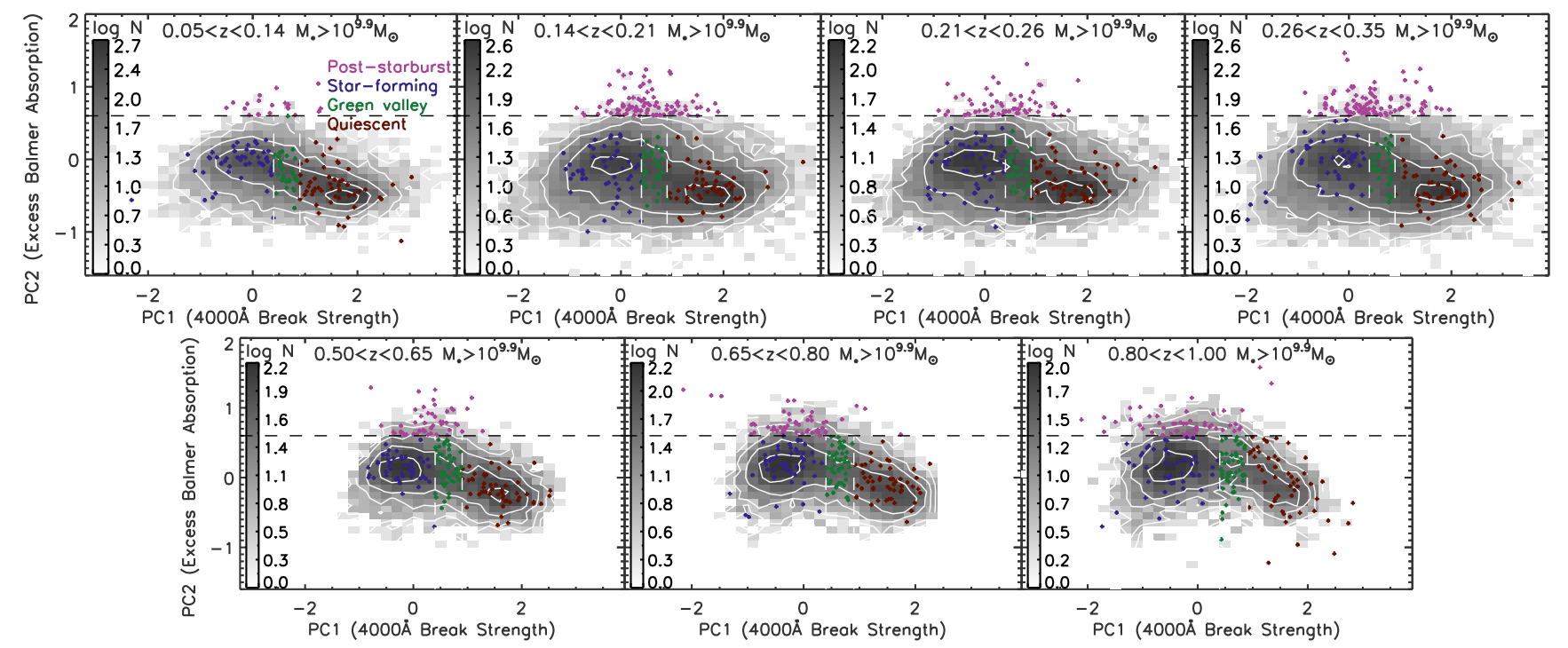

Figure 1. The distribution of the $4000 \AA$ break strength (PC1) and excess Balmer absorption (PC2) as measured by a PCA of the $4000 \AA$ spectral region of the GAMA and VIPERS galaxies, in the seven different redshift bins used in this work. The grey scale indicates the logarithmic number of objects. The coloured dots are random samples of galaxies which occupy each spectral class delineated by dashed lines: quiescent (red), star forming (blue), green valley (green) and PSB (purple); these are discussed in detail in Section 2.3. Contours show 10, 30, 50, 70 and 90 per cent of the maximum number of galaxies in the sample.

which are inside the photometric mask. 616 galaxies with broadline AGN (agnFlag $=1$ or $10<$ zflg $<20$ ) were excluded from our analysis. We restrict the upper redshift limit of the VIPERS survey to $z=1.0$, as above this redshift we are only mass complete to the most massive galaxies $\left(\mathbf{M}_{*}>10^{11.5}\right)$ which are not the main subject of this study. Our final sample comprises 29734 galaxies on which to perform spectroscopic classification.

\subsection{Spectroscopic sample classification}

In the integrated optical fibre spectrum of a galaxy, the signatures of stars of different ages can be used to obtain information about a galaxy's recent SFH. To define our sample, we make use of two particular features of optical spectra: the $4000 \AA$ break strength and Balmer absorption line strength. Following the method outlined in Wild et al. (2007, 2009), we define two spectral indices which are based on a principal component analysis (PCA) of the 3750-4150 $\AA$ region of the spectra. PC1 is the strength of the $4000 \AA$ break (equivalent to the $D_{n} 4000$ index), and PC2 is excess Balmer absorption (of all Balmer lines simultaneously) over that expected for the $4000 \AA$ break strength. The eigenbasis that defines the principal components is taken from Wild et al. (2009), and was built using observed VIMOS-VLT Deep Survey (VVDS) spectra.

To calculate the principal component amplitudes for each spectrum, we correct for Galactic extinction using the Cardelli, Clayton \& Mathis (1989) extinction law, shift to rest-frame wavelengths and interpolate the spectra on to a common wavelength grid. We then project each spectrum on to the eigenbasis using the 'gappy-PCA' procedure of Connolly \& Szalay (1999) to account for possible gaps in the spectra. Pixels are weighted by their errors during the projection, and gaps in the spectra due to bad pixels are given zero weight. The normalization of the spectra is also free to vary in the projection using the method introduced by Wild et al. (2007).

In Fig. 1, we show the distribution of the two spectral indices for galaxies in the GAMA and VIPERS surveys which have $\mathrm{S} / \mathrm{N}$ per $6 \AA$ pixel $>6.5$ at $\sim 4000 \AA$. This choice of $\mathrm{S} / \mathrm{N}$ cut allows us to reliably measure spectral indices from low-resolution spectra (Wild et al. 2009). Our sample comprises 70668 and 21519 galaxies from GAMA and VIPERS, respectively.

In Fig. 1, we divide our sample into four spectral classes based on their values of PC1 and PC2. The boundaries between the spectral classes are red: $\mathrm{PC} 1>0.9$, green: $<0.4<\mathrm{PC} 1<0.9$, star forming: $\mathrm{PC} 1<0.4$ and PSB: $\mathrm{PC} 2>0.6$. Classification is not influenced in any way by commonly used star formation indicators such as [O II] and $\mathrm{H} \alpha$ fluxes. After a starburst, the Balmer absorption lines increase in strength as the galaxy passes into the PSB phase (Dressler \& Gunn 1983; Couch \& Sharples 1987) i.e. A/F star light dominates the integrated galaxy spectrum. These objects with stronger Balmer absorption lines compared to their expected $4000 \AA$ break strength lie to the top of each panel in Fig. 1. The boundaries for the PSB class are defined to select the population outliers with high PC2. At low redshift, there are very few PSBs in each of the four redshift bins in the GAMA survey, so we collapse all of the PSBs into one large redshift bin from $0.05<z<0.26$ so that we have sufficient number statistics for our analysis (see Fig. A1). We cannot extend the PSB sample to the highest redshift range of the GAMA sample as our mass completeness drops below our 90 per cent limit (see Section 2.6) for $M_{*}>10^{10.6} M_{\odot}$. We visually inspected all of the candidate PSB spectra above our S/N limit. As shown in Appendix A, we found that $\sim 2 / 3$ of GAMA galaxies with $\mathrm{PC} 2>0.6$ are contaminants caused by problems with unmasked noise spikes, or exhibited an extreme fall-off in flux to the blue (this could be due to poor tracing of the fibre flux on the CCD when the $\mathrm{S} / \mathrm{N}$ is low). Furthermore, some spectra in the PSB region were removed if we could not positively identify a Balmer series. We note that if we did not remove the visually identified contaminants from the PSB sample our conclusions would be unchanged, even if the PSBs are twice as numerous at low redshift.

One concern is that, as we exclude broad-line AGN from our samples and PSBs are found to contain a higher fraction of narrowline AGN than other galaxies (Yan et al. 2006; Wild et al. 2007), we may be systematically missing PSBs from our samples. However, a typical AGN lifetime is two orders of magnitude shorter than the time during which PSB features are visible (e.g. Martini \& 

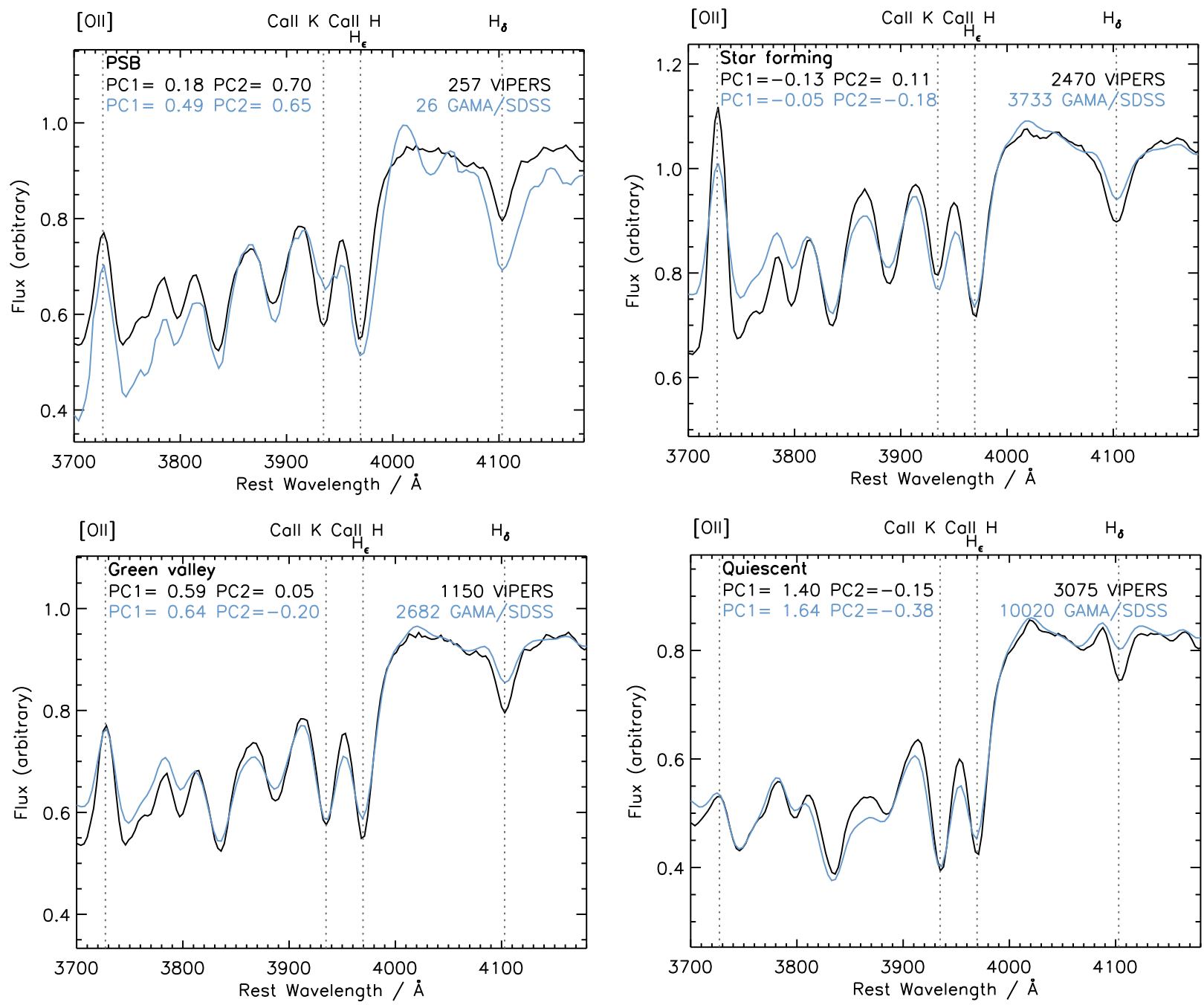

Figure 2. Stacked spectra in each spectral class with $10.6<\log _{10}\left(\mathrm{M}_{*}\right)<11$. We stack galaxies in a fixed mass range so that we can be sure we are comparing similar galaxies in each survey. Black lines show the stacked VIPERS spectra with $0.5<z<0.6$, blue lines show the stacked GAMA/SDSS spectra with $0.05<z<0.35$ convolved to the same resolution as the VIPERS spectra. Note that the PSBs are selected to be at $0.05<z<0.26$. The stacked spectra are normalized to the same value at $4000 \AA$ to aid comparison of the two samples. Dotted vertical lines indicate the rest-frame vacuum wavelengths of emission and absorption lines labelled at the top of each panel.

Weinberg 2001), thus even if all PSBs undergo a powerful unobscured AGN phase (which we consider unlikely at the redshifts studied in this paper), we will only miss a small fraction of galaxies.

Galaxies which show no evidence of recent or current star formation comprise the quiescent population which lies on the right in each panel of Fig. 1, as they have a strong $4000 \AA$ break. Galaxies that are forming stars lie in the centre and left of each panel. These galaxies have younger mean stellar ages and therefore weaker $4000 \AA$ breaks. Galaxies in the sparsely populated region between the star-forming and quiescent populations are defined as green valley (akin to that of the green valley in NUV/optical colourmagnitude diagrams). These spectroscopic green-valley galaxies do not show characteristic deep Balmer absorption lines, which indicate a slower transition for these galaxies compared to PSB galaxies. We make fixed cuts in PC1 and PC2 to separate our spectral classes, and we do not evolve these with redshift. This is because we want to select candidate transition populations between defined limits (i.e. fixed age) to test whether galaxies are changing from star forming to quiescent through these transition populations.
The boundaries between the spectral classes are somewhat arbitrary, but the broad-band colours of spectroscopically selected galaxies lie in the expected regions of the $g-r$ colour-magnitude diagram (see Figs B1 and B2). Stacking spectra in each class with similar stellar masses shows that on average the galaxies show the expected characteristic features, see Fig. 2. Star-forming galaxies show strong emission lines, a weak $4000 \AA$ break and blue continua. The stacked quiescent galaxies show strong $4000 \AA$ breaks and no emission lines. Green-valley galaxies show spectra intermediate between those of star-forming and quiescent galaxies with moderately strong $4000 \AA$ breaks and weak emission lines. PSBs have strong Balmer absorption lines and moderately strong $4000 \AA$ breaks. Our stacked PSB spectra show a strong [O II] emission line; we measure equivalent widths (EWs) of -10.8 and $-10.1 \AA$ for the stacked GAMA and VIPERS spectra, respectively. Note that our selection method makes no cuts on emission line strength, as is often done in the selection of PSBs (Goto 2005, 2007). If we were to use the Goto (2007) cut of [O II] EW $>-2.5 \AA$, the average PSB in our sample would be excluded. It is important not to exclude 
galaxies with emission lines, as narrow line AGN are common in PSB samples (Wild et al. 2007; Yan et al. 2006, 2009), and shocks can excite emission lines in PSBs (Alatalo et al. 2016a). We defer examination of the ionizing sources in PSBs to a future paper.

The stacked spectra in each spectral class look very similar in the $4000 \AA$ break region for both the GAMA/SDSS and VIPERS samples. The similarity is quantified by the values of PC1 and PC2 of the stacked spectra for the GAMA and VIPERS samples in Fig. 2. This shows that our PCA method is successful at selecting similar galaxies in each sample, despite differences in redshift and the initial spectral resolution. We observe a slightly weaker $4000 \AA$ break and stronger Balmer absorption lines in the stacked VIPERS spectra, indicating that on average the higher redshift galaxies are younger. This is most pronounced in the stacked star-forming spectra, where a significantly stronger [O $\left.\mathrm{O}_{\mathrm{II}}\right]$ line is visible in the VIPERS spectra compared to GAMA spectra consistent with the expected increase in the specific SFR of galaxies with redshift.

\subsection{Stellar masses}

Stellar masses were calculated for each galaxy using a Bayesian analysis which accounts for the degeneracy between physical parameters. Specifically, we fit a library of tens of thousands (depending on the redshift) of Bruzual \& Charlot (2003) population synthesis models to the $F U V-K$ broad-band photometry, to obtain a probability density function (PDF) for each physical property. The model libraries have a wide range of SFHs, two-component dust contents (Charlot \& Fall 2000) and metallicities from 0.5$2 \mathrm{Z}_{\odot}$. The assumed model SFHs assume a Chabrier (2003) initial mass function (IMF) and are exponentially declining with superimposed random starbursts with priors as described in Kauffmann et al. (2003). We use the median of the PDF to estimate the stellar mass and the 16th and 84th percentiles to estimate the associated uncertainty. We calculate our own stellar masses instead of using those of Taylor (2011) for consistency with the VIPERS stellar masses. When comparing our stellar mass estimates with those of Taylor (2011) we see an offset which changes with redshift; there is a 0.1 dex offset at $z=0.05$ and -0.05 dex offset at $z=0.35$. This is likely due to differences in the dust models and SFHs used in the spectral energy distribution (SED) fitting (see Wright et al. (2017) who saw similar offsets between the Taylor (2011) and their stellar masses as a function of redshift). We find good agreement between our stellar masses and those derived using the MAGPHYS code in Wright et al. (2017), with only a small 0.05 dex offset at $z=0.35$. This offset is likely because Wright et al. (2017) use observed frame $F U V-500 \mu m$ data to estimate the stellar masses and we only use $F U V-K$ magnitudes. As galaxies are dustier at high redshift, this can cause a slight shift in the stellar masses. We also compare our stellar masses to those in the MPA-JHU catalogue ${ }^{2}$ which are calculated using fits to the SDSS DR7 ugriz photometry. There is a systematic offset of 0.1 dex as a result of using different stellar population models but we do not see any trend with redshift. Performing our analysis using the Taylor (2011) stellar mass measurements does not change our conclusions. In Appendix C, we compare our mass functions to those in the literature and generally find excellent agreement for both the GAMA and VIPERS samples.

\footnotetext{
${ }^{2}$ http://wwwmpa.mpa-garching.mpg.de/SDSS/DR7/
}

\subsection{Incompleteness corrections}

We correct our number densities and mass functions for volume effects using the standard $V_{\max }$ method (Schmidt 1968), which weights the volume, $V$ (the volume out to the redshift of each galaxy) by $1 / V_{\max }$, which is the maximum volume over which a galaxy is visible in a magnitude limited survey or the upper redshift limit of a given redshift bin. It is important to account for the variety of SED shapes in a sample (Ilbert et al. 2004), as galaxies with a particular SED shape are visible out to different distances. We do this by using the best-fitting SED model found when calculating the stellar masses. We scale the best-fitting model to the observed galaxy brightness and then shift it in redshift to determine the maximum distance out to which the galaxy could be seen, given the survey magnitude limits.

As we only select spectra which have a high enough $\mathrm{S} / \mathrm{N}$ to reliably compute spectral indices, we must correct for the fraction of missing galaxies before calculating number densities. Following Wild et al. (2009), we define the quality sampling rate (QSR) as the fraction of galaxies above the $\mathrm{S} / \mathrm{N}$ threshold of 6.5 , relative to the total number of galaxies in each stellar mass bin. We compute the weight $w_{\mathrm{i}}^{\mathrm{QSR}}$ in stellar mass bins of width $0.1 \mathrm{dex}$ and redshift bins with widths of $\delta z=0.05-0.09$ for the GAMA survey, and $\delta z=0.15-0.2$ for the VIPERS data. We also multiply the QSR correction by a factor to account for the fraction of spectra which do not have a PCA measurement due to e.g. failure of the projection due to having $>20$ per cent bad pixels, which is $<1$ per cent of the total sample. The number of spectra that are missing due to fibre fringing, low-quality redshifts $(n Q<3)$ or highly uncertain PCA results is 13-33 per cent, depending on the redshift bin, and we account for this loss in our weighting scheme. We additionally account for the 5 per cent of spectra excluded from our sample which are not SDSS or GAMA spectra.

In VIPERS, only $\sim 40$ per cent of the targets meeting the selection criteria in a given field were observed. We apply a statistical weight $w_{\mathrm{i}}^{\mathrm{TSR}}$ as detailed in Guzzo et al. (2014) to correct for the fraction of photometric objects which were not targeted (the target sampling rate, TSR). In GAMA, the spectroscopic completeness is 98 percent. To correct for the missing spectra we use a TSR correction of 0.98 . The ability to securely measure a spectroscopic redshift is a function of the observing conditions, and the brightness of the target. We correct for the fraction of targeted galaxies without secure redshifts (the spectroscopic sampling rate, SSR), and perform a completeness correction due to the colour selection (the colour sampling rate, CSR). Details of the SSR and CSR are given in Guzzo et al. (2014) and Garilli et al. (2014). The GAMA sample has no colour selection criteria, so there are no SSR or CSR corrections to the low-redshift sample.

The weight given to each galaxy $\left(w_{\mathrm{i}}\right)$ is

$\frac{1}{\mathrm{~V}_{\max } \times w_{\mathrm{i}}^{\mathrm{SSR}} \times w_{\mathrm{i}}^{\mathrm{TSR}} \times w_{\mathrm{i}}^{\mathrm{CSR}} \times w_{\mathrm{i}}^{\mathrm{QSR}}}$.

In Appendix $\mathrm{C}$, we show that our corrections account for all sources of incompleteness as they allow us to recover total stellar mass functions which are consistent with published studies.

\subsection{Mass completeness limits}

The 90 per cent mass completeness limits were calculated in each redshift bin and separately for each spectral class following Pozzetti et al. (2010). It is important to do this separately for each spectral class, as our star-forming galaxy sample is complete to lower stellar 
Table 1. The single Schechter function fit parameters fitted to the total, PSB, red, green-valley and star-forming mass functions in each redshift bin. The third column shows the total number of galaxies in each class in the redshift bin. The fourth column shows the 90 per cent mass completeness limit in $\log _{10}\left(\mathrm{M}_{*} / \mathrm{M}_{\odot}\right)$ for each bin and spectroscopic class. The fifth column shows the number of galaxies in each class in the redshift bin above the mass completeness limit. Uncertainties on each parameter account for the formal fitting errors on the Schechter function, uncertainty on the stellar masses, and cosmic variance. For the green-valley galaxies in the highest redshift bin, and the PSBs in the lowest and highest redshift bins we fix $\alpha$ to -1.0 because there are not enough points to adequately constrain the faint end slope.

\begin{tabular}{|c|c|c|c|c|c|c|c|}
\hline Class & Redshift & Number & Completeness limit & Number & $\left(\phi^{\star} / \mathrm{Mpc}^{-3}\right)$ & $\log _{10}\left(\mathrm{M}_{*} / \mathrm{M}_{\odot}\right)$ & $\alpha$ \\
\hline Total & $0.05<z<0.14$ & 13696 & 10.02 & 12303 & $3.87 \pm 0.40 \times 10^{-3}$ & $10.80 \pm 0.04$ & $-0.76 \pm 0.10$ \\
\hline Total & $0.14<z<0.21$ & 22321 & 10.36 & 14756 & $2.61 \pm 0.32 \times 10^{-3}$ & $10.93 \pm 0.04$ & $-1.02 \pm 0.11$ \\
\hline Total & $0.21<z<0.26$ & 13805 & 10.59 & 8110 & $1.82 \pm 0.30 \times 10^{-3}$ & $10.97 \pm 0.05$ & $-1.12 \pm 0.17$ \\
\hline Total & $0.26<z<0.35$ & 20846 & 10.87 & 9946 & $1.71 \pm 0.22 \times 10^{-3}$ & $11.02 \pm 0.05$ & $-1.07 \pm 0.23$ \\
\hline Total & $0.50<z<0.65$ & 14091 & 10.33 & 5988 & $3.23 \pm 0.22 \times 10^{-3}$ & $10.77 \pm 0.04$ & $-0.48 \pm 0.14$ \\
\hline Total & $0.65<z<0.80$ & 13962 & 10.60 & 5037 & $3.12 \pm 0.17 \times 10^{-3}$ & $10.78 \pm 0.05$ & $-0.38 \pm 0.26$ \\
\hline Total & $0.80<z<1.00$ & 10125 & 10.87 & 2688 & $2.18 \pm 0.24 \times 10^{-3}$ & $10.86 \pm 0.09$ & $-0.73 \pm 0.50$ \\
\hline PSB & $0.05<z<0.26$ & 172 & 10.57 & 33 & $7.62 \pm 16.61 \times 10^{-5}$ & $10.23 \pm 0.27$ & $-1.00 \pm 0.00$ \\
\hline PSB & $0.50<z<0.65$ & 180 & 10.45 & 73 & $5.04 \pm 6.88 \times 10^{-5}$ & $10.81 \pm 0.53$ & $-0.99 \pm 1.50$ \\
\hline PSB & $0.65<z<0.80$ & 332 & 10.46 & 171 & $1.52 \pm 0.31 \times 10^{-4}$ & $10.58 \pm 0.23$ & $-0.37 \pm 1.07$ \\
\hline PSB & $0.80<z<1.00$ & 362 & 10.81 & 121 & $1.32 \pm 0.68 \times 10^{-4}$ & $10.86 \pm 0.11$ & $-1.00 \pm 0.00$ \\
\hline Quiescent & $0.05<z<0.14$ & 6936 & 10.00 & 6750 & $3.02 \pm 0.12 \times 10^{-3}$ & $10.65 \pm 0.03$ & $0.16 \pm 0.09$ \\
\hline Quiescent & $0.14<z<0.21$ & 9655 & 10.39 & 8383 & $2.14 \pm 0.10 \times 10^{-3}$ & $10.80 \pm 0.03$ & $-0.20 \pm 0.12$ \\
\hline Quiescent & $0.21<z<0.26$ & 5457 & 10.64 & 4365 & $1.37 \pm 0.09 \times 10^{-3}$ & $10.87 \pm 0.05$ & $-0.39 \pm 0.24$ \\
\hline Quiescent & $0.26<z<0.35$ & 8921 & 10.94 & 5823 & $1.18 \pm 0.13 \times 10^{-3}$ & $10.90 \pm 0.06$ & $-0.15 \pm 0.36$ \\
\hline Quiescent & $0.50<z<0.65$ & 2829 & 10.36 & 2648 & $1.64 \pm 0.08 \times 10^{-3}$ & $10.68 \pm 0.04$ & $0.34 \pm 0.17$ \\
\hline Quiescent & $0.65<z<0.80$ & 2493 & 10.62 & 2160 & $1.33 \pm 0.14 \times 10^{-3}$ & $10.73 \pm 0.05$ & $0.32 \pm 0.30$ \\
\hline Quiescent & $0.80<z<1.00$ & 1192 & 11.01 & 712 & $0.81 \pm 0.77 \times 10^{-3}$ & $11.10 \pm 0.25$ & $-1.79 \pm 1.08$ \\
\hline Green & $0.05<z<0.14$ & 2066 & 9.99 & 1926 & $1.04 \pm 0.06 \times 10^{-3}$ & $10.33 \pm 0.06$ & $0.21 \pm 0.26$ \\
\hline Green & $0.14<z<0.21$ & 3351 & 10.38 & 2302 & $0.90 \pm 0.19 \times 10^{-4}$ & $10.42 \pm 0.08$ & $0.16 \pm 0.53$ \\
\hline Green & $0.21<z<0.26$ & 2005 & 10.60 & 1209 & $0.66 \pm 0.12 \times 10^{-4}$ & $10.57 \pm 0.11$ & $-0.24 \pm 0.70$ \\
\hline Green & $0.26<z<0.35$ & 3039 & 10.89 & 1334 & $0.68 \pm 0.24 \times 10^{-4}$ & $10.70 \pm 0.19$ & $-0.65 \pm 1.39$ \\
\hline Green & $0.50<z<0.65$ & 960 & 10.36 & 792 & $6.64 \pm 0.57 \times 10^{-4}$ & $10.63 \pm 0.10$ & $-0.24 \pm 0.46$ \\
\hline Green & $0.65<z<0.80$ & 868 & 10.68 & 589 & $4.54 \pm 2.99 \times 10^{-4}$ & $10.58 \pm 0.15$ & $0.51 \pm 1.11$ \\
\hline Green & $0.80<z<1.00$ & 476 & 10.95 & 251 & $6.35 \pm 3.66 \times 10^{-4}$ & $10.80 \pm 0.09$ & $-1.00 \pm 0.00$ \\
\hline SF & $0.05<z<0.14$ & 4661 & 10.10 & 3060 & $2.33 \pm 0.35 \times 10^{-3}$ & $10.35 \pm 0.07$ & $-0.75 \pm 0.28$ \\
\hline SF & $0.14<z<0.21$ & 9145 & 10.34 & 3999 & $1.57 \pm 0.41 \times 10^{-3}$ & $10.57 \pm 0.09$ & $-1.26 \pm 0.34$ \\
\hline SF & $0.21<z<0.26$ & 6243 & 10.54 & 2440 & $1.23 \pm 0.48 \times 10^{-3}$ & $10.68 \pm 0.12$ & $-1.48 \pm 0.52$ \\
\hline SF & $0.26<z<0.35$ & 8712 & 10.80 & 2466 & $1.28 \pm 0.52 \times 10^{-3}$ & $10.73 \pm 0.15$ & $-1.51 \pm 0.75$ \\
\hline SF & $0.50<z<0.65$ & 3437 & 10.17 & 2150 & $1.65 \pm 0.24 \times 10^{-3}$ & $10.52 \pm 0.06$ & $-0.83 \pm 0.21$ \\
\hline SF & $0.65<z<0.80$ & 4388 & 10.43 & 1919 & $1.56 \pm 0.25 \times 10^{-3}$ & $10.64 \pm 0.08$ & $-0.83 \pm 0.34$ \\
\hline SF & $0.80<z<1.00$ & 2984 & 10.68 & 909 & $1.01 \pm 0.11 \times 10^{-3}$ & $10.66 \pm 0.11$ & $-0.42 \pm 0.63$ \\
\hline
\end{tabular}

masses than quiescent galaxies in a given redshift bin. We calculate the mass completeness limit using the stellar mass of each galaxy if it had a magnitude equal to the survey magnitude limit, so that $\log _{10}\left(\mathrm{M}_{\text {lim }}\right)=\log _{10}(\mathrm{M})+0.4\left(m-m_{\lim }\right)$, where $\mathrm{M}$ is the galaxy stellar mass, $m$ is the observed apparent magnitude in the survey selection band ( $r$ for GAMA and $i$ for VIPERS), and $m_{\text {lim }}$ is the survey magnitude limit $(r=19.8 \mathrm{mag}$ for GAMA and $i=22.5 \mathrm{mag}$ for VIPERS). We use the $\mathrm{M}_{\mathrm{lim}}$ of the faintest 20 per cent of these galaxies to represent galaxies with a typical $M / L$ ratio near the survey limit. We then calculate the 90 per cent mass completeness limit of these typical faint galaxies, assuming $\mathbf{M}_{\mathrm{lim}}$ is for galaxies in a relatively narrow redshift bin. The mass completeness limits for each spectral class and redshift bin are given in Table 1 .

\subsection{Uncertainties}

The total uncertainty in the number and stellar mass densities are calculated by adding in quadrature the errors due to sample size, uncertainty on the stellar masses, and those due to cosmic variance. The errors due to sample size (i.e. Poisson uncertainty) are estimated following Moustakas et al. (2013), where the method of Gehrels (1986) is used to compute the upper and lower limits on the uncertainty in the mass function. This method properly accounts for the uncertainty on a value when there are a small number of galaxies per mass bin, which is common at the high-mass end of the stellar mass function. We estimate the cosmic variance in each GAMA and VIPERS field with the publicly available tool GETCV (Moster et al. 2011). As the GAMA survey covers three separate fields, and VIPERS covers two separate fields, the uncertainty due to cosmic variance is reduced further, as the uncertainties for each field are combined following Moster et al. (2011). The uncertainties due to cosmic variance are minimized by the large survey volumes, and range from 5 to 11 per cent at $M_{*} \sim 10^{10.6}$ and 6 to 12 per cent at $\mathrm{M}_{*}>10^{11}$ in the GAMA survey, and 4 to 6 per cent at $\mathrm{M}_{*} \sim 10^{10.6}$ to 6 to 8 per cent at $M_{*}>10^{11}$ in the VIPERS survey. To estimate the impact of the uncertainty in stellar mass on the mass function and the cumulative number densities, we perturb each stellar mass by a random amount drawn from a Gaussian distribution with a standard deviation equal to the $1 \sigma$ error on the stellar mass. We do this for 100 realizations and take the standard deviation of the number and mass density in each stellar mass and redshift bin.

There are also systematic uncertainties in the stellar mass due to the choice of stellar population models and IMF of around $\sim 0.2$ $0.3 \mathrm{dex}$; see Wright et al. (2017) for a discussion of the effect of different stellar mass estimates on the galaxy stellar mass function. We note that we have used exactly the same method to calculate 
the stellar mass in both samples, which is crucial to make this comparison valid, therefore systematics between the two samples are minimized.

\subsection{Aperture bias}

We note that our results could be affected by aperture bias, as the fibre spectra cover a larger proportion of the galaxy at high redshift. Galaxy outskirts are usually bluer than the centre in spiral galaxies, but early-type galaxies tend to have flat or positive colour gradients (Gonzalez-Perez, Castander \& Kauffmann 2011). Since the majority of the quiescent population is likely comprised of early-type galaxies, aperture bias should have a negligible impact on our results involving the quiescent population. Indeed, both the SDSS and GAMA fibres cover $>90$ per cent of the flux from a model galaxy with an effective radius of $4 \mathrm{kpc}$ and with Sérsic index of 4 at $z>0.1$. However, for the star-forming, PSB and green-valley populations, at low redshift these may be classified as having redder spectra. We may therefore select fewer galaxies at low redshift than at high redshift, leading to an overestimation of the decline in these populations with time. The SDSS (GAMA) fibres cover 32-78 per cent (18-56 per cent) of the flux from a model galaxy with Sérsic index of 1 at $z=0.1-0.3$, respectively. We note that green-valley and PSB galaxies have a range of Sérsic indices and so will not be as affected by aperture bias as the star-forming galaxies, which tend to have lower Sérsic indices. Furthermore, Pracy et al. (2012) found that low-redshift PSBs showed declining Balmer absorption line strengths with increasing radius. At higher redshift, we may select fewer PSBs because aperture effects dilute the Balmer line strength, but the amount by which aperture bias affects the spectra of low-redshift PSBs may not be equal to the amount by which Balmer absorption bias affects the spectra of high-redshift PSBs. We test the effects of aperture bias on the classifications of galaxies in Fig. B2 and find no evidence that the broad-band colours of spectroscopically classified galaxies change with redshift. We therefore conclude that aperture bias has a negligible effect on our results.

Whilst aperture corrections are available for physical parameters such as SFR and have been shown to be robust for large galaxy populations (Brough et al. 2013; Richards et al. 2016), aperture corrections for detailed stellar population analysis are not available. This issue will be addressed by next generation integral field spectroscopic surveys such as Mapping Nearby Galaxies at APO (MaNGA, Bundy et al. 2015) and SydneyAustralian-Astronomical-Observatory Multi-object Integral-Field Spectrograph (SAMI, Croom et al. 2012; Bryant et al. 2015).

\section{RESULTS}

Most previous studies of the build-up of the number and stellar mass density of star-forming and quiescent galaxies have used broad-band data (e.g. Arnouts et al. 2007; Ilbert et al. 2013; Muzzin et al. 2013; Moustakas et al. 2013). Studies of the actively quenching populations which may be responsible for the build-up of the quiescent population have been limited to small samples of spectroscopically identified PSBs $(<20)$ at $0.5<z<1$ (Wild et al. 2009; Vergani et al. 2010), which cannot be split by mass due to small number statistics (although see Pattarakijwanich et al. 2016 who select $\sim 6000$ PSBs from the SDSS at $0.05<z<1.3$ ). We use our spectroscopic classifications of a large sample of quiescent, star-forming, green-valley and PSB galaxies to see how each of the populations change as a function of stellar mass over a wide redshift range from $0.05<z<1.0$. In the following analysis, we only use redshift bins above the 90 per cent mass completeness limit.

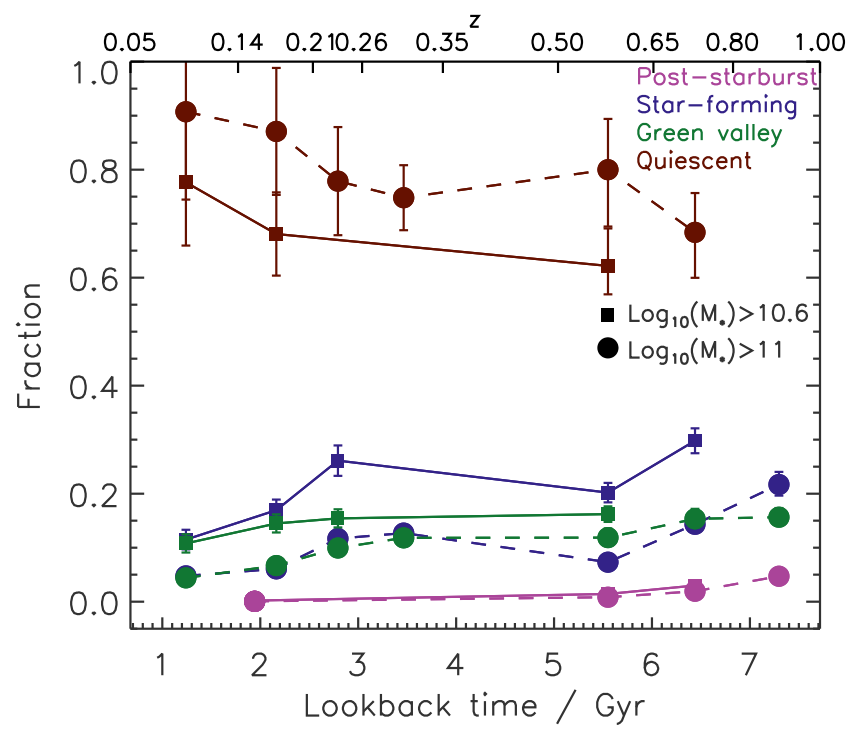

Figure 3. The volume-corrected fraction of quiescent, star-forming, greenvalley and PSB galaxies as a function of stellar mass and redshift. Intermediate-mass galaxies with $\mathrm{M}_{*}>10^{10.6} \mathrm{M}_{\odot}$ are shown as small squares joined with solid lines, and high-mass galaxies with $\mathbf{M}_{*}>10^{11} \mathbf{M}_{\odot}$ are shown as large circles joined with dashed lines. Uncertainties are propagated from the number densities and include Poisson errors, cosmic variance and those due to uncertainties on the stellar mass, which are typically smaller than the symbol size.

\subsection{Fractions in each spectral class}

In Fig. 3, we show the fraction of quiescent, star-forming, greenvalley and PSB galaxies in each mass bin as a function of redshift. As a function of stellar mass, $>60$ percent of intermediate-mass $\left(\mathrm{M}_{*}>10^{10.6} \mathrm{M}_{\odot}\right.$; small squares $)$ and $>70$ percent of high-mass $\left(\mathrm{M}_{*}>10^{11} \mathrm{M}_{\odot}\right.$; large circles) galaxies are in the quiescent population, and high-mass star-forming galaxies are rare $(<30$ per cent and $<25$ per cent for intermediate- and high-mass galaxies, respectively). The quiescent fraction increases from high to low redshift, while the star-forming fraction is decreasing towards low redshift, independent of stellar mass. The fraction of galaxies in the green valley at intermediate masses $\left(\mathrm{M}_{*}>10^{10.6} \mathrm{M}_{\odot}\right.$; small squares $)$ is $\sim 16$ percent at $z=0.6$, decreasing slightly to 11 percent at $z=0.1$. The fraction of high-mass green-valley galaxies decreases more steeply from 15 per cent to 4 per cent from $z=0.7$ to 0.1 . The PSB galaxies are rare at any redshift, and comprise only $0.06-0.2$ per cent of the total galaxy population at $z<0.35$, rising to $2-3$ per cent of the population at $z \sim 0.7$, depending on stellar mass. Our rising PSB fraction with redshift is in agreement with the findings of Dressler et al. (2013) and Wild et al. (2009, 2016). The PSBs are rare compared to green-valley galaxies, this may be because they are only visible for a short time, whereas galaxies in the green valley may spend longer there, as we discuss in the following sections.

\subsection{Number density evolution}

We present the completeness corrected cumulative number densities of spectroscopically identified star-forming, quiescent, green-valley and PSB galaxies as a function of redshift and mass in Table 2 and Fig. 4. If we move the spectral classification boundaries by $\Delta(\mathrm{PC})=0.1$ (twice as large as the typical uncertainty on PC1 and PC2) then the quiescent, star-forming and green-valley number densities change a negligible amount, but the PSB number densities 
Table 2. The cumulative comoving log number densities $\left(\mathrm{Mpc}^{-3}\right)$ of galaxies in each spectroscopic class in redshift bins above a given stellar mass limit. Values are only shown for bins above the 90 per cent mass completeness limit in each spectroscopic class. Uncertainties include Poisson errors, cosmic variance and those due to uncertainties on the stellar mass.

\begin{tabular}{|c|c|c|c|c|c|}
\hline Redshift & All & Star forming & PSB & Quiescent & Green \\
\hline \multicolumn{6}{|c|}{$\log _{10}\left(\mathrm{M} / \mathrm{M}_{*}\right)>10.6$} \\
\hline $0.05<z<0.14$ & $-2.75_{-0.05}^{+0.04}$ & $-3.69_{-0.05}^{+0.05}$ & $-5.63_{-0.08}^{+0.08}$ & $-2.86_{-0.05}^{+0.04}$ & $-3.72_{-0.05}^{+0.05}$ \\
\hline $0.14<z<0.21$ & $-2.79_{-0.04}^{+0.03}$ & $-3.56_{-0.04}^{+0.03}$ & $-5.63_{-0.08}^{+0.08}$ & $-2.95_{-0.04}^{+0.03}$ & $-3.63_{-0.04}^{+0.03}$ \\
\hline $0.21<z<0.26$ & $-2.91_{-0.03}^{+0.03}$ & $-3.50_{-0.03}^{+0.03}$ & $-5.63_{-0.08}^{+0.08}$ & - & $-3.72_{-0.04}^{+0.03}$ \\
\hline $0.26<z<0.35$ & - & - & - & - & - \\
\hline $0.50<z<0.65$ & $-2.85_{-0.03}^{+0.03}$ & $-3.54_{-0.03}^{+0.03}$ & $-4.70_{-0.06}^{+0.05}$ & $-3.06_{-0.03}^{+0.03}$ & $-3.64_{-0.03}^{+0.03}$ \\
\hline $0.65<z<0.80$ & $-2.85_{-0.02}^{+0.02}$ & $-3.37_{-0.03}^{+0.02}$ & $-4.37_{-0.04}^{+0.04}$ & - & - \\
\hline $0.80<z<1.00$ & - & - & - & - & - \\
\hline \multicolumn{6}{|c|}{$\log _{10}\left(\mathrm{M} / \mathrm{M}_{*}\right)>11$} \\
\hline $0.05<z<0.14$ & $-3.41_{-0.06}^{+0.05}$ & $-4.73_{-0.10}^{+0.09}$ & $-6.60_{-0.34}^{+0.24}$ & $-3.45_{-0.06}^{+0.05}$ & $-4.76_{-0.10}^{+0.09}$ \\
\hline $0.14<z<0.21$ & $-3.38_{-0.04}^{+0.04}$ & $-4.59_{-0.06}^{+0.05}$ & $-6.60_{-0.34}^{+0.24}$ & $-3.44_{-0.04}^{+0.04}$ & $-4.55_{-0.06}^{+0.05}$ \\
\hline $0.21<z<0.26$ & $-3.48_{-0.04}^{+0.04}$ & $-4.41_{-0.05}^{+0.05}$ & $-6.60_{-0.34}^{+0.24}$ & $-3.59_{-0.04}^{+0.04}$ & $-4.48_{-0.05}^{+0.05}$ \\
\hline $0.26<z<0.35$ & $-3.39_{-0.03}^{+0.02}$ & $-4.29_{-0.03}^{+0.03}$ & $-6.60_{-0.34}^{+0.24}$ & $-3.52_{-0.03}^{+0.02}$ & $-4.32_{-0.03}^{+0.03}$ \\
\hline $0.50<z<0.65$ & $-3.44_{-0.04}^{+0.03}$ & $-4.58_{-0.07}^{+0.06}$ & $-5.53_{-0.19}^{+0.14}$ & $-3.54_{-0.04}^{+0.03}$ & $-4.37_{-0.05}^{+0.05}$ \\
\hline $0.65<z<0.80$ & $-3.38_{-0.03}^{+0.03}$ & $-4.23_{-0.05}^{+0.04}$ & $-5.10_{-0.10}^{+0.08}$ & $-3.55_{-0.03}^{+0.03}$ & $-4.20_{-0.04}^{+0.04}$ \\
\hline $0.80<z<1.00$ & $-3.49_{-0.03}^{+0.03}$ & $-4.16_{-0.04}^{+0.04}$ & $-4.82_{-0.06}^{+0.05}$ & - & $-4.30_{-0.04}^{+0.04}$ \\
\hline
\end{tabular}

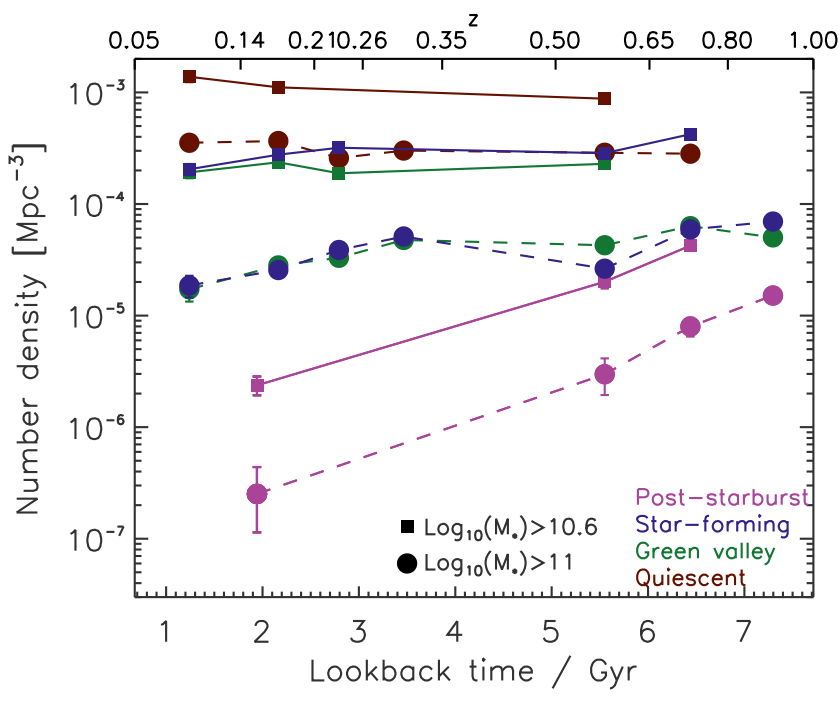

Figure 4. The completeness corrected comoving number densities $\left(\mathrm{Mpc}^{-3}\right)$ for star-forming, red, green-valley and PSB galaxies in redshift and stellar bins. Intermediate-mass galaxies with $\mathrm{M}_{*}>10^{10.6} \mathrm{M}_{\odot}$ are shown as small squares joined with solid lines, and high-mass galaxies with $\mathrm{M}_{*}>10^{11} \mathrm{M}_{\odot}$ are shown as large circles joined with dashed lines. Points are only shown for bins above the 90 per cent completeness limit. Errors include uncertainties due to sample size and cosmic variance.

change by a factor of 2 . The trends that we observe with redshift remain unchanged. Qualitatively our results are robust to changes in the spectral classification boundaries and stellar mass binning.

For intermediate- $\left(\mathrm{M}_{*}>10^{10.6} \mathrm{M}_{\odot}\right)$ and high-mass $\left(\mathrm{M}_{*}>10^{11} \mathrm{M}_{\odot}\right)$ star-forming galaxies, the population declines in number density between $z=0.6$ and 0.1 . For intermediate-mass $\left(\mathrm{M}_{*}>10^{10.6} \mathrm{M}_{\odot}\right)$ quiescent galaxies, the population grows in number density by a factor of 1.58 between $z=0.6$ and 0.1 . We find that the number density of high-mass $\left(\mathrm{M}_{*}>10^{11} \mathrm{M}_{\odot}\right)$ quiescent galaxies increases by a factor of 1.23 between $z=0.6$ and 0.1 . Our results are similar to those of Moustakas et al. (2013), who found that the number density of quiescent galaxies (selected using a cut in the broad-band photometry-derived $\mathrm{M}_{*}-\mathrm{SFR}$ relation) grows more slowly for high-mass galaxies from $z=1$ to 0.1 . We cannot recover the number density of less massive $\left(\mathrm{M}_{*}<10^{10} \mathrm{M}_{\odot}\right)$ galaxies beyond the lowest redshift bin as our sample becomes incomplete in low-mass galaxies at $z>0.14$. Deeper spectroscopy or the use of photometric galaxy classification methods (Wild et al. 2014) are still required to probe the low stellar mass quiescent galaxy regime. It may be that the quiescent population is growing more rapidly at low redshift than suggested by our results, but only at lower masses than those probed by our study (Tinker et al. 2013; Muzzin et al. 2013).

To date, there have been few studies of the number densities of candidate transition (green-valley and PSB) galaxies. We find that green-valley galaxies with intermediate masses of $\mathbf{M}_{*}>10^{10.6} \mathrm{M}_{\odot}$ have an approximately flat number density of $\sim 10^{-3.7} \mathrm{Mpc}^{-3}$ from $z=1$ to 0 . At high stellar masses $\left(\mathrm{M}_{*}>10^{11} \mathrm{M}_{\odot}\right)$, the number density of green-valley galaxies decreases by an order of magnitude from $z=1$ to 0 .

The number density of intermediate-mass $\left(\mathrm{M}_{*}>10^{10.6} \mathrm{M}_{\odot}\right)$ and high-mass $\left(\mathrm{M}_{*}>10^{11} \mathrm{M}_{\odot}\right)$ PSB galaxies decreases by an order of magnitude in the redshift range $0.2<z<0.6$. Our results are qualitatively consistent with the results of Wild et al. (2016) who found a factor of 3 decrease in the number density of $\mathrm{M}_{*}>10^{10.6} \mathrm{M}_{\odot}$ PSBs from $z=2$ to 0.5 (see also Dressler et al. 2013). Our results also agree with the lower limits on the number density of compact, massive $\left(M_{*}>10^{11} M_{\odot}\right) \mathrm{E}+\mathrm{A}$ galaxies at $0.2<z<0.8$ from Zahid et al. (2016). Using VVDS spectra, Wild et al. (2009) found that there are more galaxies passing through the PSB phase at high redshift than at low redshift. The number densities of intermediatemass PSBs in our study at $0.5<z<0.65$ are similar to those in Wild et al. (2016), who found a number density of $10^{-4.9} \mathrm{Mpc}^{-3}$ for $\mathrm{M}_{*}>10^{10.5} \mathrm{M}_{\odot}$ PSBs at $0.5<z<1$. However, the PSB number density at $0.65<z<0.8$ is larger than that of Wild et al. (2016). This discrepancy may be because Wild et al. (2016) use a photometric selection method which may not be as sensitive to PSB features as 
Table 3. The cumulative comoving log mass densities $\left(\mathrm{M}_{\odot} \mathrm{Mpc}^{-3}\right)$ of galaxies in each spectroscopic class in redshift bins above a given stellar mass limit. Values are only shown for bins above the 90 per cent mass completeness limit in each spectroscopic class. Uncertainties include Poisson errors, cosmic variance and those due to uncertainties on the stellar mass.

\begin{tabular}{|c|c|c|c|c|c|}
\hline Redshift & All & Star forming & PSB & Quiescent & Green \\
\hline \multicolumn{6}{|c|}{$\log _{10}\left(\mathrm{M} / \mathrm{M}_{*}\right)>10.6$} \\
\hline $0.05<z<0.14$ & $8.17_{-0.05}^{+0.04}$ & $7.15_{-0.06}^{+0.05}$ & $5.30_{-0.11}^{+0.09}$ & $8.08_{-0.05}^{+0.04}$ & $7.09_{-0.05}^{+0.05}$ \\
\hline $0.14<z<0.21$ & $8.16_{-0.04}^{+0.03}$ & $7.28_{-0.04}^{+0.04}$ & $5.30_{-0.11}^{+0.09}$ & $8.03_{-0.04}^{+0.03}$ & $7.22_{-0.04}^{+0.03}$ \\
\hline $0.21<z<0.26$ & $8.04_{-0.03}^{+0.03}$ & $7.36_{-0.03}^{+0.03}$ & $5.30_{-0.11}^{+0.09}$ & - & $7.16_{-0.04}^{+0.03}$ \\
\hline $0.26<z<0.35$ & - & - & - & - & - \\
\hline $0.50<z<0.65$ & $8.09_{-0.03}^{+0.03}$ & $7.27_{-0.03}^{+0.03}$ & $6.15_{-0.06}^{+0.05}$ & $7.93_{-0.03}^{+0.03}$ & $7.25_{-0.03}^{+0.03}$ \\
\hline $0.65<z<0.80$ & $8.11_{-0.02}^{+0.02}$ & $7.47_{-0.03}^{+0.03}$ & $6.50_{-0.04}^{+0.04}$ & - & - \\
\hline $0.80<z<1.00$ & - & - & - & - & - \\
\hline \multicolumn{6}{|c|}{$\log _{10}\left(\mathrm{M} / \mathrm{M}_{*}\right)>11$} \\
\hline $0.05<z<0.14$ & $7.80_{-0.06}^{+0.05}$ & $6.59_{-0.11}^{+0.09}$ & $4.94_{-0.34}^{+0.24}$ & $7.75_{-0.06}^{+0.05}$ & $6.37_{-0.10}^{+0.08}$ \\
\hline $0.14<z<0.21$ & $7.84_{-0.04}^{+0.04}$ & $6.69_{-0.06}^{+0.05}$ & $4.94_{-0.34}^{+0.24}$ & $7.77_{-0.04}^{+0.04}$ & $6.63_{-0.06}^{+0.05}$ \\
\hline $0.21<z<0.26$ & $7.73_{-0.04}^{+0.04}$ & $6.81_{-0.05}^{+0.05}$ & $4.94_{-0.34}^{+0.24}$ & $7.63_{-0.04}^{+0.04}$ & $6.69_{-0.05}^{+0.05}$ \\
\hline $0.26<z<0.35$ & $7.84_{-0.03}^{+0.02}$ & $6.89_{-0.03}^{+0.03}$ & $4.94_{-0.34}^{+0.24}$ & $7.73_{-0.03}^{+0.02}$ & $6.84_{-0.03}^{+0.03}$ \\
\hline $0.50<z<0.65$ & $7.74_{-0.04}^{+0.03}$ & $6.54_{-0.07}^{+0.06}$ & $5.60_{-0.19}^{+0.14}$ & $7.66_{-0.04}^{+0.03}$ & $6.78_{-0.05}^{+0.05}$ \\
\hline $0.65<z<0.80$ & $7.81_{-0.03}^{+0.03}$ & $6.91_{-0.05}^{+0.04}$ & $6.02_{-0.11}^{+0.09}$ & $7.66_{-0.03}^{+0.03}$ & $6.96_{-0.04}^{+0.04}$ \\
\hline $0.80<z<1.00$ & $7.71_{-0.03}^{+0.03}$ & $7.00_{-0.04}^{+0.03}$ & $6.37_{-0.06}^{+0.05}$ & - & $6.89_{-0.04}^{+0.04}$ \\
\hline
\end{tabular}

the spectroscopic selection used in our study. The number density of PSB galaxies identified spectroscopically from the VVDS survey with $0.5<z<1.0$ by Wild et al. (2009) was $10^{-4} \mathrm{Mpc}^{-3}$ for galaxies with $\mathbf{M}_{*}>10^{9.75} \mathbf{M}_{\odot}$, measured from 16 PSB galaxies. As we are highly incomplete at such low stellar masses, we cannot directly compare to the results from Wild et al. (2009), which used VVDS data which are two magnitudes deeper than the VIPERS survey.

The stellar mass densities (Table 3 ) show very similar behaviour to the number densities. For intermediate-mass $\left(\mathrm{M}_{*}>10^{10.6} \mathrm{M}_{\odot}\right)$ quiescent galaxies, the population grows in stellar mass density by a factor of 1.41 between $z=0.6$ and 0.1 . The mass density of high-mass $\left(\mathrm{M}_{*}>10^{11} \mathrm{M}_{\odot}\right)$ quiescent galaxies grows by a factor of 1.23 between $z=0.6$ and 0.1 . Our results for the growth of the quiescent population are smaller than those of Bell et al. (2004), Brown et al. (2007) and Arnouts et al. (2007), who found that the quiescent population has doubled in mass in the range $0<z<1$. The differences between our measured mass growth rate and literature studies may be because our spectroscopic selection, stellar mass and redshift range are slightly different to those in other studies. Furthermore, we have checked that aperture bias does not cause us to misclassify large numbers of galaxies at low redshift, see Fig. B2. Our results show that, in general, there were more transition galaxies at high redshift than in the local Universe.

\subsection{Evolution of mass functions}

We present the mass functions of the red, star-forming, green-valley and PSB galaxies in Fig. 5. We fit our mass functions with single Schechter functions (Schechter 1976), using the IMPRO IDL library ${ }^{3}$ (Moustakas et al. 2013). We do not fit our mass functions with double Schechter functions as we do not see an upturn at low stellar masses. The Schechter fit parameters are in Table 1. The uncertainties on the number density $(\phi)$ include contributions from Poisson errors, cosmic variance and from uncertainties on the stel-

\footnotetext{
${ }^{3}$ https://github.com/moustakas/impro
}

lar mass estimated via a Monte Carlo method with 100 realizations (Section 2.7).

The mass functions of the quiescent galaxies show a clear buildup in the low-mass end from $z=1$ to 0 , and a smaller increase in the number density of high-mass galaxies, as is commonly found in the literature. Conversely, the mass functions of the star-forming galaxies show that from $z=1$ to 0 , there is a decline in the number density of massive galaxies with redshift. Note that our spectroscopically defined quiescent population mass function is different to that selected on $u-r$ and optical colour from Baldry et al. (2012, also using GAMA data), as we find fewer low-mass galaxies (see Appendix C). This may be because Baldry et al. (2012) separate star-forming and quiescent galaxies using broad-band colours, and we use a cleaner spectroscopic selection that likely has less contamination by dusty objects. Furthermore, we separate quiescent from green-valley galaxies, whereas Baldry et al. (2012) do not make this discrimination, meaning that green-valley galaxies will be mixed with the red and blue populations defined with broad-band colours (Taylor et al. 2015). See Appendix B for an analysis of the broad-band colours of galaxies in each spectral class.

As seen in Section 3.2, the green-valley galaxy mass functions show a negligible build-up at $\mathrm{M}_{*} \sim 10^{10.5} \mathrm{M}_{\odot}$ with redshift, but there is more evolution at the high-mass end of the mass function. There were more high-mass galaxies in the green valley at high redshift than at low redshift. The PSB mass function exhibits stronger evolution in the mass function than green-valley galaxies, with galaxies in the PSB phase more massive at high redshift. Our transition galaxy mass functions are consistent with more massive galaxies quenching earlier, and less massive galaxies quenching later. Similar results were found by Gonçalves et al. (2012) for green-valley galaxies.

\section{HOW FAST DO GALAXIES QUENCH?}

Previous studies using broad-band photometry have not reached a consensus on the relative importance of the fast and slow channels for galaxy quenching and building of the quiescent galaxy 

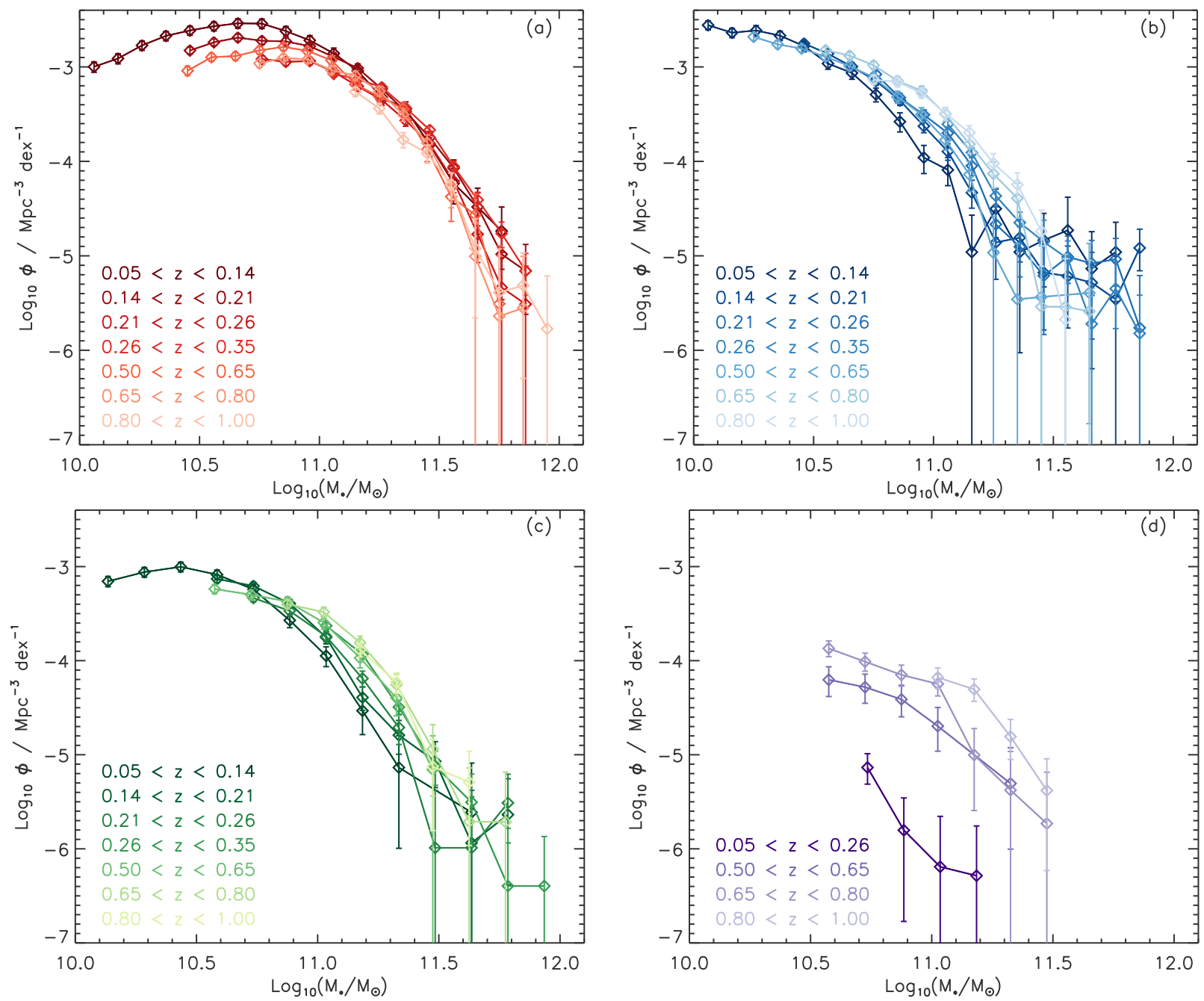

Figure 5. The stellar mass functions (corrected for incompleteness) for (a) quiescent, (b) star-forming, (c) green-valley and (d) PSBs, in different redshift bins. Points are only shown where the bin is $>90$ per cent complete in stellar mass. Errors include Poisson uncertainties, cosmic variance and from uncertainties on the stellar masses.

population. Spectroscopic surveys offer the unique advantage of being able to cleanly identify both quiescent galaxies and candidate transition galaxies with different quenching time-scales. In this section, we discuss our results in terms of the relative importance of these two channels for building the quiescent population.

\subsection{Quiescent galaxies}

We showed in Section 3.2 that the number density of the quiescent population is consistent with slow evolution at $0.05<z<1$ for $\mathbf{M}_{*}>10^{10.6} \mathbf{M}_{\odot}$, and almost flat evolution for $\mathbf{M}_{*}>10^{11} \mathrm{M}_{\odot}$. We estimate the rate at which galaxies are entering the quiescent population $(\mathrm{d} \phi / \mathrm{d} t)$ by fitting a straight line to the number density as a function of time to each mass bin of the quiescent population. The quantity $\mathrm{d} \phi / \mathrm{d} t$ is shown for intermediate- $\left(\mathrm{M}_{*}>10^{10.6} \mathrm{M}_{\odot}\right)$ and high-mass $\left(\mathrm{M}_{*}>10^{11} \mathrm{M}_{\odot}\right)$ quiescent galaxies in Fig. 6 as the solid red and dashed lines, respectively. The hashed areas represent the upper and lower limits on the growth rate of the quiescent population, derived from the uncertainty on the linear fit to the quiescent population number densities as a function of time. While it is possible that the growth rate is not linear with time, the current data do not allow any higher order terms to be fit. In the following discussion, we neglect the effect of dry mergers which would cause quiescent galaxies to move within mass bins, as at these high masses the rate of dry mergers with close to equal mass ratios is expected to be small. While high-mass quiescent galaxies may merge with low-mass companions, they are not thought to merge with each other at low redshift to sufficiently affect their number density.

For the intermediate-mass $\left(\mathrm{M}_{*}>10^{10.6} \mathrm{M}_{\odot}\right)$ quiescent galaxies, we find a number density evolution with time which is growing at a rate of $\mathrm{d} \phi / \mathrm{d} t=8.8 \pm 2.5 \times 10^{-5} \mathrm{Mpc}^{-3} \mathrm{Gyr}^{-1}$. For the highmass $\left(\mathrm{M}_{*}>10^{11} \mathrm{M}_{\odot}\right)$ quiescent galaxies, we find a growth rate of $\mathrm{d} \phi / \mathrm{d} t=7.6 \pm 6.7 \times 10^{-6} \mathrm{Mpc}^{-3} \mathrm{Gyr}^{-1}$. We note that aperture effects could cause us to classify galaxies differently depending on their redshift, particularly in the lowest redshift bin. We test the robustness of our results to redshift effects by repeating the growth rate calculation without the lowest redshift point and measure a slightly slower growth rate in both mass bins. However, the values are consistent within the $1 \sigma$ uncertainties and our conclusions remain unchanged.

\subsection{Green-valley galaxies}

To put an upper limit on the rate at which green-valley galaxies could be passing into the quiescent population $(\mathrm{d} \phi / \mathrm{d} t)$, we divide 


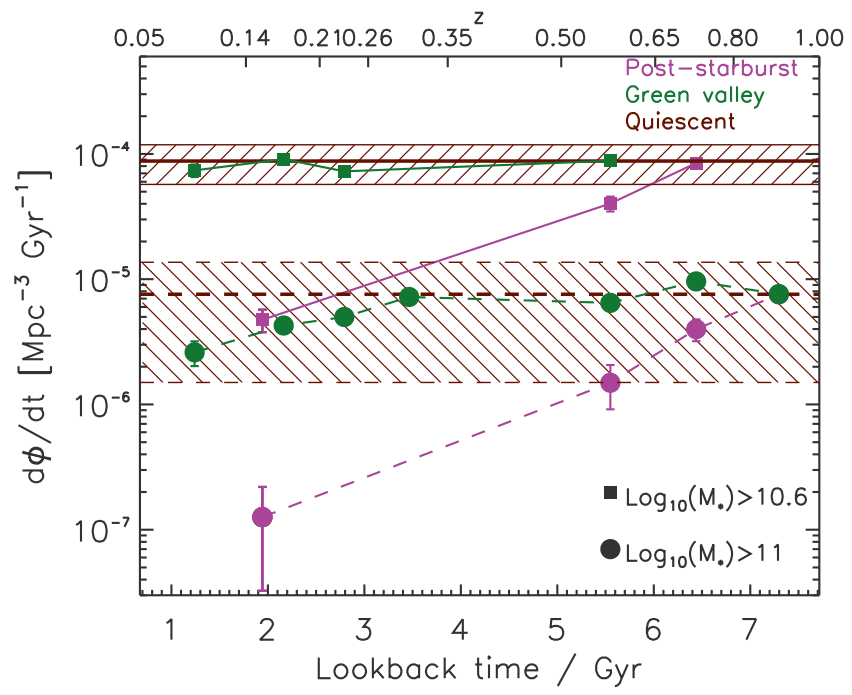

Figure 6. The rate of galaxies passing through the PSB (purple) and greenvalley (green) phase, and the growth rate of the quiescent population, as a function of redshift and mass. We assume a nominal transition time-scale of 2.6 and $6.6 \mathrm{Gyr}$ for intermediate- and high-mass green-valley galaxies, respectively (see Section 4.2), and 0.5 and 2.0 Gyr for intermediate- and high-mass PSBs, respectively (see Section 4.3). Transition time-scales are estimated by requiring that $\mathrm{d} \phi / \mathrm{d} t_{\text {Greenvalley }} \leq \mathrm{d} \phi / \mathrm{d} t_{\text {Quiescent }}$ and $\mathrm{d} \phi / \mathrm{d} t_{\text {PSB }}$ $\leq \mathrm{d} \phi / \mathrm{d} t_{\text {Quiescent }}$ in the highest redshift bin. Results for intermediate-mass $\left(\mathrm{M}_{*}>10^{10.6} \mathrm{M}_{\odot}\right)$ galaxies are shown as small squares connected by solid lines; high-mass $\left(\mathrm{M}_{*}>10^{11} \mathrm{M}_{\odot}\right)$ galaxies are shown as large circles connected by dashed lines. The errors for the PSB and green-valley populations include Poisson, cosmic variance contributions and uncertainties propagated from the uncertainty on the stellar masses. The growth rate of the quiescent population is shown as a thick red line. The hatched area represents the uncertainty on the growth rate of the quiescent population, which is derived from the uncertainty on the linear fit to the number densities. The growth rate of star-forming galaxies of all masses is negative and is not shown.

the number densities in the intermediate-mass bin by a nominal transition time-scale such that $\mathrm{d} \phi / \mathrm{d} t_{\text {Greenvalley }} \leq \mathrm{d} \phi / \mathrm{d} t_{\text {Quiescent }}$. This corresponds to a lower limit on the transition time-scale. To do this, we make the assumptions that (1) galaxies cannot be transitioning faster than the growth rate of the quiescent population, and (2) that PSBs do not contribute to the growth of the quiescent population. In reality both green-valley and PSB populations may be transitioning into the quiescent population, which would then require longer transition time-scales than given here. We discuss this further below. Green-valley galaxies with $\mathrm{M}_{*}>10^{10.6} \mathrm{M}_{\odot}$ are transitioning at a rate of $8 \times 10^{-5} \mathrm{Mpc}^{-3} \mathrm{Gyr}^{-1}$, for a transition time-scale of $2.6 \mathrm{Gyr}$. Because the number density of intermediatemass green-valley galaxies remains constant with cosmic time, this transition time-scale refers to the rate at which galaxies pass through the boundaries we have defined for the green valley, assuming that every galaxy in this region is transitioning. If the transition timescale does not change with redshift, the observed flat number density leads to a flat transition rate, which is consistent with a linear growth rate for the quiescent population. Similar conclusions of a relatively unchanging green-valley population were found by Salim et al. (2012), Fang et al. (2012) and Salim (2014) who studied the UV morphologies and SFHs of green-valley galaxies.

The estimated transition time-scale of $2.6_{-0.7}^{+1.4} \mathrm{Gyr}$ (accounting for the uncertainty on the growth rate of the quiescent population) is entirely reasonable, and is similar to time-scales found in the literature. Accounting for uncertainties, the transition time-scale for green-valley galaxies cannot be $>4 \mathrm{Gyr}$ otherwise $\mathrm{d} \phi / \mathrm{d} t_{\text {Greenvalley }}$ will exceed $\mathrm{d} \phi / \mathrm{d} t_{\text {Quiescent }}$. Using cosmological hydrodynamical simulations with radiation transfer post-processing, Trayford et al. (2016) found using broad-band colours that most simulated galaxies spend $<2 \mathrm{Gyr}$ in the green valley, independent of galaxy mass. Martin et al. (2007) used spectral indices to estimate quenching times in local green-valley galaxies, finding a time-scale of $50 \mathrm{Myr}$ to $6 \mathrm{Gyr}$, with more than 50 per cent of quenching occurring within 2 Gyr. Using broad-band colours, Smethurst et al. (2015) found a continuum of transition time-scales, but that most galaxies spend $1-2$ Gyr in the green valley. Differences in transition time-scales may be due to the differences in selection methods of green-valley galaxies (e.g. optical or UV colour-magnitude, spectroscopy).

In Fig. 6, we show $\mathrm{d} \phi / \mathrm{d} t$ for high-mass $\left(\mathrm{M}_{*}>10^{11} \mathrm{M}_{\odot}\right)$ greenvalley galaxies, assuming a transition time-scale of $6.6_{-2.9}^{+26.6} \mathrm{Gyr}$. Given the large errors on the growth of the high-mass quiescent population, the drop in number density of the high-mass greenvalley galaxies remains consistent with a transition time-scale that does not evolve with redshift. If we assumed a shorter time-scale of 2.6 Gyr as used for the intermediate-mass galaxies, the rate at which high-mass galaxies pass through the green valley would be formally inconsistent with the growth rate of the quiescent population, after accounting for uncertainties at the $1 \sigma$ level. Our findings hint at an increasing transition time-scale with increasing mass, and may explain our slightly longer time-scale compared to other published values given our high-mass limit $\left(\mathrm{M}_{*}>10^{10.6} \mathrm{M}_{\odot}\right)$.

Our results show that the growth of the quiescent population at these masses and redshifts can be entirely explained by galaxies transitioning slowly through the green valley. The presence of another, faster quenching channel would require a longer transition time for green-valley galaxies, or alternatively that only a fraction of the galaxies are actually transitioning.

\subsection{Post-starburst galaxies}

We can put an upper limit on the rate at which galaxies could be passing through the PSB phase and into the quiescent population in the same way as for the green-valley galaxies. To reconcile the rate of intermediate-mass $\left(\mathrm{M}_{*}>10^{10.6} \mathrm{M}_{\odot}\right)$ galaxies transitioning through the PSB phase at $z=0.7$ with the growth rate of the quiescent population, we find a transition time-scale of $0.5 \mathrm{Gyr}$. As above, this assumes that the green-valley galaxies do not contribute to the growth of the quiescent population.

A transition time-scale of $0.5_{-0.1}^{+0.3} \mathrm{Gyr}$ (accounting for the uncertainty of the growth rate of the quiescent population) is a reasonable estimate for PSB galaxies and is similar to the time-scales found in hydrodynamical merger simulations by Wild et al. (2009), where the simulations are observed with the same spectral indices as used to identify PSB galaxies in this paper. Visibility time-scales of a few hundred Myr for PSB features were also found in similar merger simulations by Snyder et al. (2011). Both papers found that the time-scales depend sensitively on gas fractions, orbital dynamics and progenitor types. The rapid decline in number density of PSB galaxies means that they must have significantly shorter visibility time-scales at low redshift if they are to contribute significantly to the growth of the quiescent population. While the simulations suggest that a visibility time-scale a factor of 2 shorter may be reasonable, this does not come close to the factor of 18 decrease in number density for intermediate-mass galaxies. Equally, while it is possible that the rate of growth of the quiescent population slows between $z=1$ and 0.05 , and this is not captured by our linear fit, 
the change in number density of quiescent galaxies does not seem to indicate such a significant change. Aperture bias may cause us to select fewer transition galaxies at low redshift than at high redshift, but our tests in Section 2.8 and Appendix B show that this is unlikely to cause such a strong evolution in number density as we observe. We can therefore conclude that while the fast-quenching PSB channel may contribute significantly to quiescent population growth of intermediate-mass galaxies at $z=0.7$, it appears to be insignificant by $z \sim 0$.

To reconcile the rate of high-mass $\left(\mathrm{M}_{*}>10^{11} \mathrm{M}_{\odot}\right)$ galaxies transitioning through the PSB phase at $z=0.7$ with the growth rate of the quiescent population, we find a transition time-scale of $2.0_{-0.9}^{+8.0} \mathrm{Gyr}$. This seems marginally inconsistent with the maximum possible visibility time-scale for PSB galaxies of $\sim 1 \mathrm{Gyr}$ (the main-sequence lifetime of A-stars). Decreasing the time-scale to a more reasonable $0.5 \mathrm{Gyr}$ would give a transition rate that is incompatible with the observed growth of the quiescent population, especially when additional growth via the green valley is included. This indicates that at high masses some PSB galaxies may not be transitioning into the quiescent population. Further processes may be needed to fully quench these high-mass transition galaxies, i.e. they will subsequently return to the green-valley or star-forming population. Alternatively, PSB galaxies may have rejuvenated from the quiescent population rather than transitioning from the star-forming population. The former scenario fits well with the findings that PSBs still have substantial gas and dust contents (Zwaan et al. 2013; French et al. 2015; Rowlands et al. 2015; Alatalo et al. 2016b), and often have discy morphologies (Pawlik et al. 2016), indicating that they may not be fully quenched.

\subsection{Are galaxies quenching?}

The growth in number density of quiescent galaxies shows that quenching is occurring at $0.05<z<1$. The data are consistent with a quenching rate that is constant with cosmic time, and when combined with the observed number density of green-valley galaxies, fits with a scenario in which the predominant quenching channel is the slow transitioning of green-valley galaxies into the quiescent population over a time-scale of $\sim 2.6 \mathrm{Gyr}$ for galaxies with $\mathrm{M}_{*}>10^{10.6} \mathrm{M}_{\odot}$, increasing to $\sim 6.6 \mathrm{Gyr}$ for galaxies with $\mathbf{M}_{*}>10^{11} \mathrm{M}_{\odot}$. The existence of a significant number of PSB galaxies at $z \sim 0.7$ draws this conclusion into question however. If both green-valley and PSB galaxies contribute to the growth of the quiescent population at $z \sim 0.7$ then the transition time-scales of both populations must be longer than the values estimated above. The maximum possible time-scale for PSB galaxies is $\sim 1 \mathrm{Gyr}$ (the main-sequence lifetime of A-stars). Therefore, at $z \sim 0.7$ the growth of the intermediate-mass quiescent population could conceivably be composed of, for example an equal fraction of PSBs with a transition time of $1 \mathrm{Gyr}$ and green-valley galaxies with a transition time of $\sim 5.2$ Gyr. Although transition time-scales are generally expected to be shorter at higher redshift (Gonçalves et al. 2012; Tinker et al. 2013; Balogh et al. 2016), better data would be required to determine the exact rate at which the quiescent population is growing with redshift in order to rule out this scenario.

However, at high masses $\left(\mathbf{M}_{*}>10^{11} \mathbf{M}_{\odot}\right)$, there is clear tension between the number density of transition galaxies and small growth rate of the quiescent population. The transition times for the high-mass galaxies are already long (2 Gyr for PSBs and 6.6 Gyr for green-valley galaxies). If both green-valley and PSB galaxies contribute to the growth of the quiescent population at $z \sim 0.7$, then the transition time-scales of both populations will be unphysically long. The large number of high-mass transition galaxies compared to the slow growth of the quiescent population could be resolved if either (i) the uncertainties in the mass growth of the quiescent population are underestimated, (ii) galaxies do not follow the linear evolutionary path of star forming, to quenching, to quiescent. The first scenario could be due to underestimation of cosmic variance, aperture bias, or systematics in the stellar masses due to IMF variation with redshift or galaxy mass. The first scenario will likely only be solved with larger spectroscopic surveys. Even though aperture bias may affect some classifications, the difference in the number densities between transition and quiescent galaxies is so large, and the evolution in the number densities of the green-valley and PSB galaxies is so strong that some misclassified galaxies are unlikely to affect our conclusions. Regarding the second scenario, several authors have suggested that PSB and green-valley galaxies may have been rejuvenated and have temporarily come out of the quiescent population (Cortese \& Hughes 2009; Fang et al. 2012; Dressler et al. 2013), and up to 60 per cent of local early-type galaxies have a cold interstellar medium (ISM, e.g. Oosterloo et al. 2010; Young et al. 2011; Serra et al. 2012; Rowlands et al. 2012; Smith et al. 2012; Agius et al. 2013) which should allow them to rejuvenate given some trigger event. The broad-band colours of these gasrich early-type galaxies are consistent with a rejuvenation scenario (Young et al. 2014), where gas has been accreted recently via minor mergers (Davis et al. 2011). Using cosmological hydrodynamical simulations with radiation transfer post-processing, Trayford et al. (2016) found that 10 percent of simulated galaxies are classified as rejuvenated as they show blue broad-band colours but were red in the past, although only 1.6 per cent of simulated galaxies rapidly change colour from red to blue over a $<2 \mathrm{Gyr}$ time period. However, Furlong et al. (2015) showed that the passive fraction is too low in EAGLE at $\log \left(\mathrm{M}_{*}>10.5\right)$ by $\sim 15$ per cent which may be a result of too much rejuvenation in their simulations. Direct comparisons of observations with simulations via the forward modelling of the simulations as mock data sets may help to unpick these related problems. A temporary departure of galaxies from the quiescent population into the PSB or green-valley phase, possibly as a result of minor merger-driven star formation, would relieve the tension between the number of candidate transition galaxies and the slow growth of the quiescent population at high masses. At intermediate masses, rejuvenation may happen, but is not visible compared to the number of truly quenching galaxies.

Alternatively, given the presence of a large cold ISM in PSBs (Zwaan et al. 2013; Rowlands et al. 2015; French et al. 2015; Alatalo et al. 2016a), high-mass galaxies may originate from, and return to, the star-forming population or green valley after a starburst. This was suggested by Dressler et al. (2013) as the starburst galaxy population far outnumbers the passive galaxies in field and group environments. Overall, our findings for the highest mass $\left(\mathrm{M}_{*}>10^{11} \mathrm{M}_{\odot}\right)$ galaxies are in agreement with Dressler et al. (2013) that the slow change in the numbers of quiescent galaxies since $z \sim 1$ indicates that either not many galaxies in the PSB phase or green valley finally join the quiescent population, or that quenching takes multiple events happening over a long time.

Ultimately, since the growth rate of the quiescent population at $\mathbf{M}_{*}>10^{10.6} \mathrm{M}_{\odot}$ is slow, there is not a lot of room for the complete quenching of all massive galaxies since $z=1$, over half the age of the Universe. This means that although most galaxies are reducing their SFRs with time, very few of them completely halt their star formation to become quiescent. At the highest masses $\left(\mathrm{M}_{*}>10^{11} \mathrm{M}_{\odot}\right)$, both rapid and slower quenching processes (e.g. 
strangulation and starvation) may be less effective. This implies that some fraction of populations commonly thought to be moving from star forming to quiescent, such as green valley and PSBs, may not be transitioning at all.

\section{SUMMARY}

By exploiting the highly complete, wide-area GAMA and VIPERS spectroscopic surveys, we have studied the number densities of quiescent, PSB and green-valley galaxies. This has allowed us to explore the rate at which galaxies are quenching at $0.05<z<1$ as a function of mass, and the contribution of different transition galaxy populations to the build-up of the quiescent population. Our main conclusions are summarized as follows:

(i) Over the last 8 billion years, the quiescent population grows in number and mass density more quickly for intermediate-mass $\left(\mathrm{M}_{*}>10^{10.6} \mathrm{M}_{\odot}\right)$ galaxies compared to high-mass galaxies $\left(\mathrm{M}_{*}>\right.$ $\left.10^{11} \mathrm{M}_{\odot}\right)$.

(ii) The number densities of spectroscopically classified greenvalley galaxies from $0<z<1$ is flat for intermediate mass and declining for high masses. We find the transition time-scale of intermediate- (high) mass green-valley galaxies is $2.6 \mathrm{Gyr}$ (6.6 Gyr) at $0.05<z<1$ if green-valley galaxies contribute 100 per cent to the growth of the quiescent population.

(iii) The number densities of PSB galaxies from $0<z<1$ is declining for both intermediate- and high-mass populations. The high-mass PSB population shows a steeper decline in number density than at intermediate masses. We find that the transition timescale of intermediate- (high) mass PSBs is $0.5 \mathrm{Gyr}(2.0 \mathrm{Gyr})$ at $z \sim 0.7$ if PSB galaxies contribute 100 per cent to the growth of the quiescent population. The rapid decline in number density of PSBs with decreasing redshift means that the fast quenching channel must be insignificant by $z \sim 0$.

(iv) If high redshift, intermediate-mass PSB galaxies are visible for a slightly longer maximum time-scale of $\sim 1 \mathrm{Gyr}$, corresponding to the main-sequence lifetime of A-stars, this allows quenching via both a slow and fast route to contribute to the growth of the intermediate-mass quiescent population at $z \sim 0.7$.

(v) Both the green-valley and PSB mass functions show that high-mass galaxies transitioned at earlier cosmic times. The PSB mass function shows stronger redshift evolution than that of the green-valley galaxy mass function.

(vi) The number of high-mass $\left(\mathrm{M}_{*}>10^{11} \mathrm{M}_{\odot}\right)$ green-valley and PSB galaxies is in tension with the observed slow growth of the quiescent population. This indicates that at high masses, some PSB or green-valley galaxies are not transitioning from the star forming into the quiescent population. The mechanisms which cause a complete shutdown in star formation may be rare or ineffective at $z<1$. Quiescent galaxies may undergo rejuvenation events which temporarily cause a galaxy to be observed in the green-valley or PSB phase. Alternatively, the tension could be eased if the uncertainties on the number densities due to cosmic variance or stellar masses are underestimated.

This study has put upper limits on the rate at which galaxies are quenching, and by how much the slow- and fast-quenching channels may be contributing at masses $\mathrm{M}_{*}>10^{10.6} \mathrm{M}_{\odot}$ and redshifts in the range $0.05<z<1$. Larger spectroscopic surveys are needed to ascertain which quenching processes are acting in different environment and mass regimes. This issue will be addressed by the Taipan survey (da Cunha 2017), which will measure fibre spectra for 1 million galaxies at $z<0.3$. Detailed information about the SFHs of candidate transition galaxies from high $\mathrm{S} / \mathrm{N}$ spectra would confirm the fraction of the progenitors that are truly coming from the star-forming population, rather than rejuvenating. The processes which transform galaxies may leave different imprints on the motion and spatial distribution of stars and gas. Integral field spectroscopy from surveys such as MaNGA and SAMI could also help us to determine which processes cause quenching as a function of mass and environment.

\section{ACKNOWLEDGEMENTS}

We thank the referee for insightful and detailed comments which improved the paper. We thank Alice Mortlock, Omar Almaini, Tim Davis and Alan Dressler for useful discussions. VW and KR acknowledge support from the European Research Council Starting Grant SEDmorph (PI: VW). GAMA is a joint EuropeanAustralasian project based around a spectroscopic campaign using the Anglo-Australian Telescope. The GAMA input catalogue is based on data taken from the SDSS and the UKIRT Infrared Deep Sky Survey. Complementary imaging of the GAMA regions is being obtained by a number of independent survey programs including GALEX MIS, VST KIDS, VISTA VIKING, WISE, Herschel-ATLAS, GMRT and ASKAP providing UV to radio coverage. GAMA is funded by the STFC (UK), the ARC (Australia), the AAO and the participating institutions. The GAMA website is http://www.gama-survey.org/. Based on observations made with ESO Telescopes at the La Silla Paranal Observatory under programme ID 179.A-2004. This paper uses data from the VIMOS Public Extragalactic Redshift Survey (VIPERS). VIPERS has been performed using the ESO Very Large Telescope, under the 'Large Programme' 182.A-0886. The participating institutions and funding agencies are listed at http://vipers.inaf.it This research has made use of NASA's Astrophysics Data System Bibliographic Services.

\section{REFERENCES}

Adelman-McCarthy J. K. et al., 2008, ApJS, 175, 297

Agius N. K. et al., 2013, MNRAS, 431, 1929

Alatalo K. et al., 2016a, ApJS, 224, 38

Alatalo K. et al., 2016b, ApJ, 827, 106

Arnouts S. et al., 2007, A\&A, 476, 137

Baldry I. K., Glazebrook K., Brinkmann J., Ivezić Ž., Lupton R. H., Nichol R. C., Szalay A. S., 2004, ApJ, 600, 681

Baldry I. K. et al., 2012, MNRAS, 421, 621

Balogh M. L. et al., 2016, MNRAS, 456, 4364

Barnes J. E., Hernquist L., 1996, ApJ, 471, 115

Barro G. et al., 2013, ApJ, 765, 104

Bell E. F. et al., 2004, ApJ, 608, 752

Benson A. J., Bower R. G., Frenk C. S., Lacey C. G., Baugh C. M., Cole S., 2003, ApJ, 599, 38

Bessell M. S., 1990, PASP, 102, 1181

Blake C. et al., 2004, MNRAS, 355, 713

Blanton M. R. et al., 2003, ApJ, 594, 186

Brammer G. B. et al., 2011, ApJ, 739, 24

Brough S. et al., 2013, MNRAS, 435, 2903

Brown M. J. I., Dey A., Jannuzi B. T., Brand K., Benson A. J., Brodwin M., Croton D. J., Eisenhardt P. R., 2007, ApJ, 654, 858

Bruzual G., Charlot S., 2003, MNRAS, 344, 1000

Bryant J. J. et al., 2015, MNRAS, 447, 2857

Bundy K. et al., 2015, ApJ, 798, 7

Cardelli J. A., Clayton G. C., Mathis J. S., 1989, ApJ, 345, 245

Chabrier G., 2003, PASP, 115, 763

Charlot S., Fall S. M., 2000, ApJ, 539, 718

Connolly A. J., Szalay A. S., 1999, AJ, 117, 2052 
Cortese L., Hughes T. M., 2009, MNRAS, 400, 1225

Couch W. J., Sharples R. M., 1987, MNRAS, 229, 423

Croom S. M. et al., 2012, MNRAS, 421, 872

Crossett J. P., Pimbblet K. A., Jones D. H., Brown M. J. I., Stott J. P., 2017, MNRAS, 464, 480

da Cunha E., 2017, preprint (arXiv:1706.01246)

Davidzon I. et al., 2013, A\&A, 558, A23

Davis T. A. et al., 2011, MNRAS, 417, 882

Di Matteo T., Springel V., Hernquist L., 2005, Nature, 433, 604

Dressler A., Gunn J. E., 1983, ApJ, 270, 7

Dressler A., Oemler Jr A., Poggianti B. M., Gladders M. D., Abramson L., Vulcani B., 2013, ApJ, 770, 62

Driver S. P. et al., 2011, MNRAS, 413, 971

Driver S. P. et al., 2016, MNRAS, 455, 3911

Faber S. M. et al., 2007, ApJ, 665, 265

Fang J. J., Faber S. M., Salim S., Graves G. J., Rich R. M., 2012, ApJ, 761, 23

Fang J. J., Faber S. M., Koo D. C., Dekel A., 2013, ApJ, 776, 63

French K. D., Yang Y., Zabludoff A., Narayanan D., Shirley Y., Walter F., Smith J.-D., Tremonti C. A., 2015, ApJ, 801, 1

Fritz A. et al., 2014, A\&A, 563, A92

Furlong M. et al., 2015, MNRAS, 450, 4486

Garilli B. et al., 2014, A\&A, 562, A23

Gehrels N., 1986, ApJ, 303, 336

Gonzalez-Perez V., Castander F. J., Kauffmann G., 2011, MNRAS, 411, 1151

Gonçalves T. S., Martin D. C., Menéndez-Delmestre K., Wyder T. K., Koekemoer A., 2012, ApJ, 759, 67

Gordon Y. A. et al., 2017, MNRAS, 465, 2671

Goto T., 2005, MNRAS, 357, 937

Goto T., 2007, MNRAS, 381, 187

Gunn J. E., Gott III J. R., 1972, ApJ, 176, 1

Guzzo L. et al., 2014, A\&A, 566, A108

Hahn C., Tinker J. L., Wetzel A. R., 2016, ApJ, 841, 17

Haines C. P. et al., 2013, ApJ, 775, 126

Hill D. T. et al., 2011, MNRAS, 412, 765

Hopkins P. F., Bundy K., Hernquist L., Ellis R. S., 2007, ApJ, 659, 976

Hopkins A. M. et al., 2013, MNRAS, 430, 2047

Iglesias-Páramo J. et al., 2013, A\&A, 553, L7

Ilbert O. et al., 2004, MNRAS, 351, 541

Ilbert O. et al., 2013, A\&A, 556, A55

Kauffmann G. et al., 2003, MNRAS, 341, 33

Kaviraj S., Kirkby L. A., Silk J., Sarzi M., 2007, MNRAS, 382, 960

Kaviraj S., Schawinski K., Silk J., Shabala S. S., 2011, MNRAS, 415, 3798

Kewley L. J., Jansen R. A., Geller M. J., 2005, PASP, 117, 227

Khalatyan A., Cattaneo A., Schramm M., Gottlöber S., Steinmetz M., Wisotzki L., 2008, MNRAS, 387, 13

Liske J. et al., 2015, MNRAS, 452, 2087

Martig M., Bournaud F., Teyssier R., Dekel A., 2009, ApJ, 707, 250

Martin D. C. et al., 2007, ApJS, 173, 342

Martini P., Weinberg D. H., 2001, ApJ, 547, 12

McCarthy I. G., Frenk C. S., Font A. S., Lacey C. G., Bower R. G., Mitchell

N. L., Balogh M. L., Theuns T., 2008, MNRAS, 383, 593

McGee S. L., Bower R. G., Balogh M. L., 2014, MNRAS, 442, L105

Mihos J. C., Hernquist L., 1994, ApJ, 431, L9

Mihos J. C., Hernquist L., 1996, ApJ, 464, 641

Mortlock A. et al., 2013, MNRAS, 433, 1185

Moster B. P., Somerville R. S., Newman J. A., Rix H.-W., 2011, ApJ, 731, 113

Moustakas J. et al., 2013, ApJ, 767, 50

Moutard T. et al., 2016, A\&A, 590, A102

Muzzin A. et al., 2013, ApJ, 777, 18

Oosterloo T. et al., 2010, MNRAS, 409, 500

Pattarakijwanich P., Strauss M. A., Ho S., Ross N. P., 2016, ApJ, 833, 19

Pawlik M. M., Wild V., Walcher C. J., Johansson P. H., Villforth C., Row-

lands K., Mendez-Abreu J., Hewlett T., 2016, MNRAS, 456, 3032

Peng Y., Maiolino R., Cochrane R., 2015, Nature, 521, 192

Peng Y.-j. et al., 2010, ApJ, 721, 193

Pozzetti L. et al., 2010, A\&A, 523, A13
Pracy M. B., Owers M. S., Couch W. J., Kuntschner H., Bekki K., Briggs F., Lah P., Zwaan M., 2012, MNRAS, 420, 2232

Richards S. N. et al., 2016, MNRAS, 455, 2826

Robotham A. S. G. et al., 2013, MNRAS, 431, 167

Rowlands K. et al., 2012, MNRAS, 419, 2545

Rowlands K., Wild V., Nesvadba N., Sibthorpe B., Mortier A., Lehnert M., da Cunha E., 2015, MNRAS, 448, 258

Salim S., 2014, Serb. Astron. J., 189, 1

Salim S., Fang J. J., Rich R. M., Faber S. M., Thilker D. A., 2012, ApJ, 755, 105

Saunders W. et al., 2004, in Moorwood A. F. M., Iye M., eds, Proc. SPIEConf. Ser. Vol. 5492, Ground-based Instrumentation for Astronomy. SPIE, Bellingham, p. 389

Schawinski K. et al., 2014, MNRAS, 440, 889

Schechter P., 1976, ApJ, 203, 297

Schlegel D. J., Finkbeiner D. P., Davis M., 1998, ApJ, 500, 525

Schmidt M., 1968, ApJ, 151, 393

Schneider D. P. et al., 2007, AJ, 134, 102

Serra P. et al., 2012, MNRAS, 422, 1835

Sharp R. et al., 2006, in McLean I. S., Iye M., eds, Proc. SPIE Conf. Ser. Vol. 6269, Ground-based and Airborne Instrumentation for Astronomy. SPIE, Bellingham, p. $62690 \mathrm{G}$

Smethurst R. J. et al., 2015, MNRAS, 450, 435

Smith D. J. B. et al., 2012, MNRAS, 427, 703

Snyder G. F., Cox T. J., Hayward C. C., Hernquist L., Jonsson P., 2011, ApJ, 741,77

Springel V., Di Matteo T., Hernquist L., 2005, ApJ, 620, L79

Strateva I. et al., 2001, AJ, 122, 1861

Sutherland W. et al., 2015, A\&A, 575, A25

Taylor E. N., 2011, MNRAS, 418, 1587

Taylor E. N. et al., 2015, MNRAS, 446, 2144

Tinker J. L., Leauthaud A., Bundy K., George M. R., Behroozi P., Massey R., Rhodes J., Wechsler R. H., 2013, ApJ, 778, 93

Tokunaga A. T., Simons D. A., Vacca W. D., 2002, PASP, 114, 180

Tomczak A. R. et al., 2014, ApJ, 783, 85

Trayford J. W., Theuns T., Bower R. G., Crain R. A., Lagos C. d. P., Schaller M., Schaye J., 2016, MNRAS, 460, 3925

Vergani D. et al., 2010, A\&A, 509, A42

Wetzel A. R., Tinker J. L., Conroy C., van den Bosch F. C., 2013, MNRAS, 432,336

Whitaker K. E., Kriek M., van Dokkum P. G., Bezanson R., Brammer G., Franx M., Labbé I., 2012, ApJ, 745, 179

Whitaker K. E. et al., 2015, ApJ, 811, L12

Wijesinghe D. B. et al., 2012, MNRAS, 423, 3679

Wild V., Kauffmann G., Heckman T., Charlot S., Lemson G., Brinchmann J., Reichard T., Pasquali A., 2007, MNRAS, 381, 543

Wild V., Walcher C. J., Johansson P. H., Tresse L., Charlot S., Pollo A., Le Fèvre O., de Ravel L., 2009, MNRAS, 395, 144

Wild V. et al., 2014, MNRAS, 440, 1880

Wild V., Almaini O., Dunlop J., Simpson C., Rowlands K., Bowler R., Maltby D., McLure R., 2016, MNRAS, 463, 832

Williams R. J., Quadri R. F., Franx M., van Dokkum P., Labbé I., 2009, ApJ, 691,1879

Wright A. H. et al., 2016, MNRAS, 460, 765

Wright A. H. et al., 2017, MNRAS, 470, 283

Wuyts S. et al., 2011, ApJ, 742, 96

Yan R., Newman J. A., Faber S. M., Konidaris N., Koo D., Davis M., 2006, ApJ, 648, 281

Yan R. et al., 2009, MNRAS, 398, 735

Yesuf H. M., Faber S. M., Trump J. R., Koo D. C., Fang J. J., Liu F. S., Wild V., Hayward C. C., 2014, ApJ, 792, 84

York D. G. et al., 2000, AJ, 120, 1579

Young L. M. et al., 2011, MNRAS, 414, 940

Young L. M. et al., 2014, MNRAS, 444, 3408

Zahid H. J., Baeza Hochmuth N., Geller M. J., Damjanov I., Chilingarian I. V., Sohn J., Salmi F., Hwang H. S., 2016, ApJ, 831, 146

Zwaan M. A., Kuntschner H., Pracy M. B., Couch W. J., 2013, MNRAS, 432,492 


\section{APPENDIX A: REMOVAL OF CONTAMINANTS FROM THE PSB POPULATION}

In Fig. A1, we show the PCA selection of candidate PSBs $(\mathrm{PC} 2>0.6)$ with $\mathrm{S} / \mathrm{N}>6.5$ in the redshift range $0.05<z<0.26$. Orange points show spectra which had problems with unmasked noise spikes, or exhibited an extreme fall-off in flux to the blue (this could be due to poor tracing of the flux on the CCD when the $S / N$ is low). Furthermore, some spectra in the PSB region were removed if we could not positively identify a Balmer series. The contaminant sources mostly occupy regions above the quiescent population and to the upper left of the star-forming galaxies, with unphysical principal component amplitudes. Around $\sim 2 / 3$ of candidate PSB galaxies were removed, leaving a clean PSB sample (purple points),

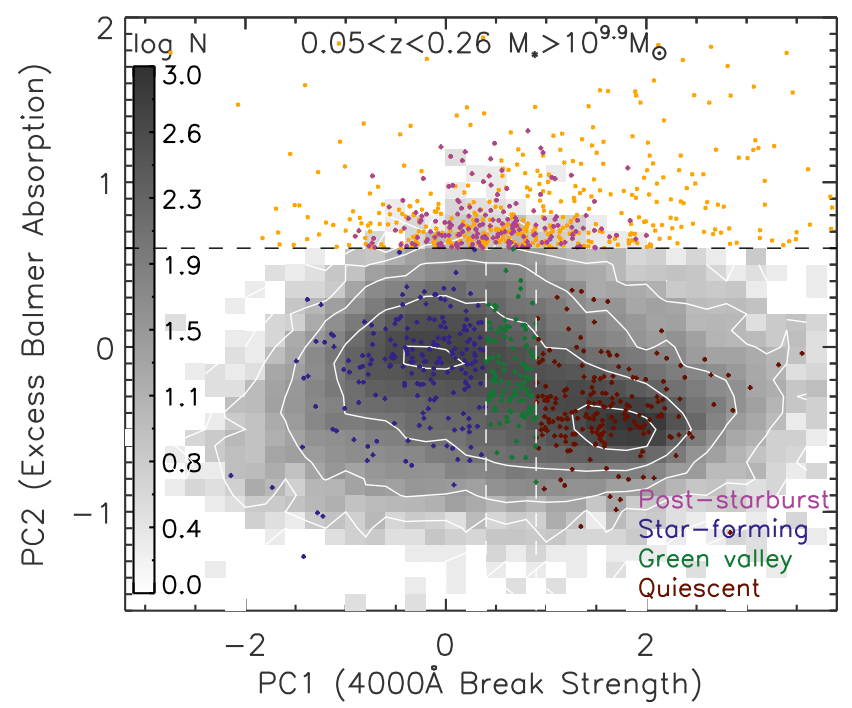

Figure A1. The distribution of the $4000 \AA$ break strength (PC1) and excess Balmer absorption (PC2) as measured using a PCA of the $4000 \AA$ spectral region of the GAMA galaxies in the range $0.05<z<0.26$. The grey scale indicates the logarithmic number of objects. The coloured dots are random samples of galaxies which occupy each spectral class delineated by dashed lines: quiescent (red), star forming (blue), green valley (green) and clean PSB (purple), rejected PSB (orange). Contours show 10, 30, 50, 70 and 90 per cent of the maximum number of galaxies in the sample. which lie in the expected PSB region above the star-forming and green-valley populations.

\section{APPENDIX B: BROAD-BAND COLOURS OF SPECTRALLY CLASSIFIED GALAXIES}

In Fig. B1, we show the $g-r$ colours of galaxies in each spectral class. The broad-band colours have been K-corrected to rest-frame wavelengths using the best-fitting model SED from the Bruzual \& Charlot (2003) library and have been corrected for Galactic extinction. The broad-band colours of PSB galaxies are mostly in the $g-r$ blue cloud and green valley, with a minority of galaxies on the red sequence. The spectroscopically classified quiescent galaxies mostly reside in the red sequence, but there is some scatter towards the green valley. The spectroscopically classified green-valley galaxies are centred on the $g-r$ green valley, but there is also substantial scatter into the blue cloud and red sequence. The spectroscopically classified star-forming galaxies lie mostly in the $g-r$ blue cloud but there is substantial scatter into the red sequence. We note that the definition of green valley based on broad-band photometry is arbitrary. The disagreement between the spectroscopic and photometric classifications for some galaxies may be because of the different effects of dust on broad-band photometry and the information from performing the PCA on the spectra, which span a relatively narrow wavelength range of 3750-4150 Å. Furthermore, aperture bias will cause some differences, as the broad-band colours are an average over the whole galaxy, but the fibre only covers $2-3 \operatorname{arcsec}$ of the galaxy centre. This is discussed further in Section 2.8.

Aperture bias is an issue which could affect our conclusions. To test this, in Fig. B2, we show the $U-V$ and $V-J$ colours (e.g. Williams et al. 2009) of the four spectral classes in four redshift bins. We compute the rest-frame $U, V$ and $J$ magnitudes by convolving the best-fitting SED model (see Section 2.4) with the Bessel $U$, Bessel $V$ (Bessell 1990) and UKIRT $J$ filters (Tokunaga, Simons \& Vacca 2002). Spectroscopically, classified star-forming galaxies lie in the blue part of the $U V J$ diagram, whilst quiescent galaxies have red $U V J$ colours. Spectroscopically, classified green-valley galaxies have intermediate colours. The majority of PSBs overlap with the star-forming and green-valley populations. Galaxies in our sample have broad-band colours consistent with their spectroscopic classes, at all redshifts. If aperture bias was affecting our results

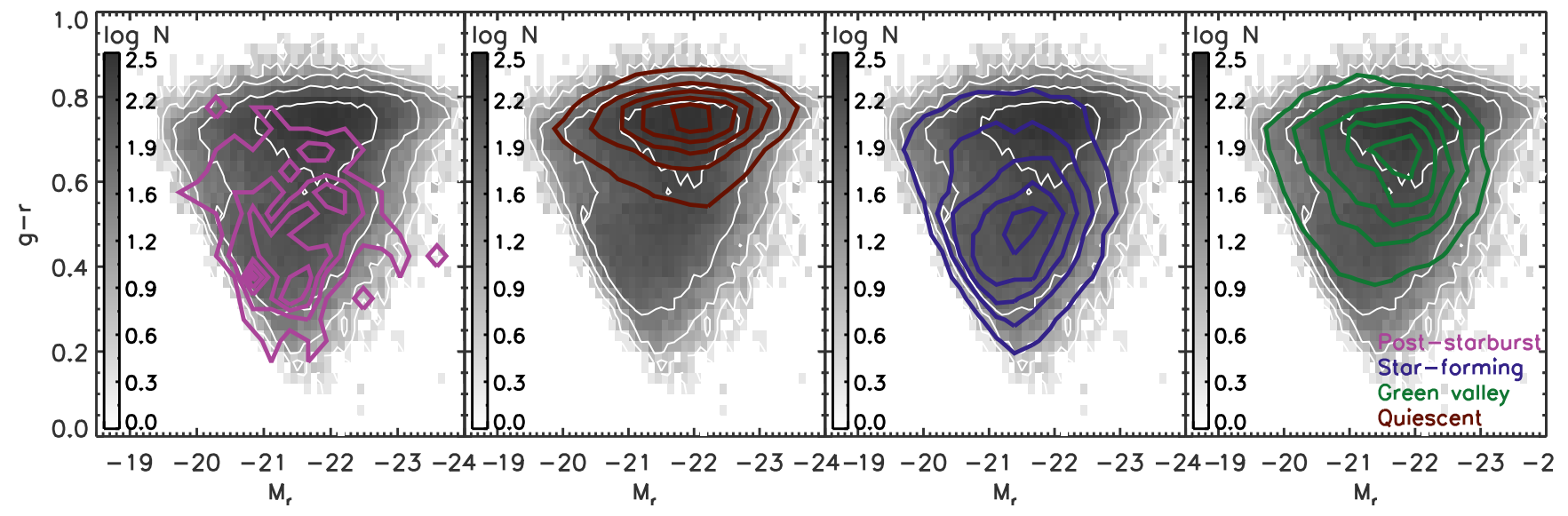

Figure B1. Rest-frame broad-band colours as a function of absolute $r$ band magnitude of spectrally classified galaxies in the GAMA survey. The grey scale indicates the logarithmic number of objects in the parent sample with $\mathrm{S} / \mathrm{N}>6.5$ per $6 \AA$ A pixel. Coloured contours show the different spectral classes in each panel, from left to right these are: PSB, quiescent, star-forming and green-valley galaxies. Contours show 10, 30, 50, 70 and 90 per cent of the maximum number of galaxies in each sample. 


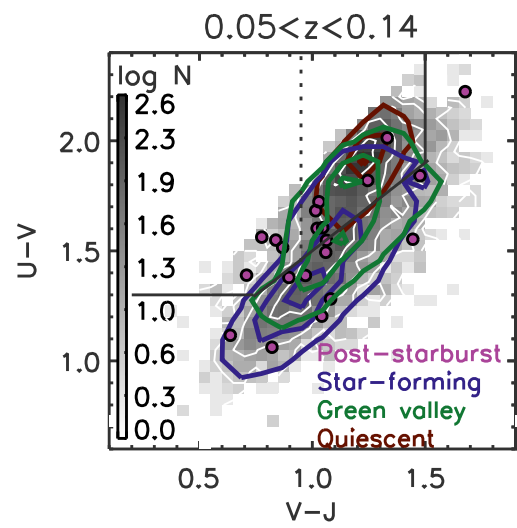

$$
0.14<z<0.21
$$

$0.26<z<0.35$
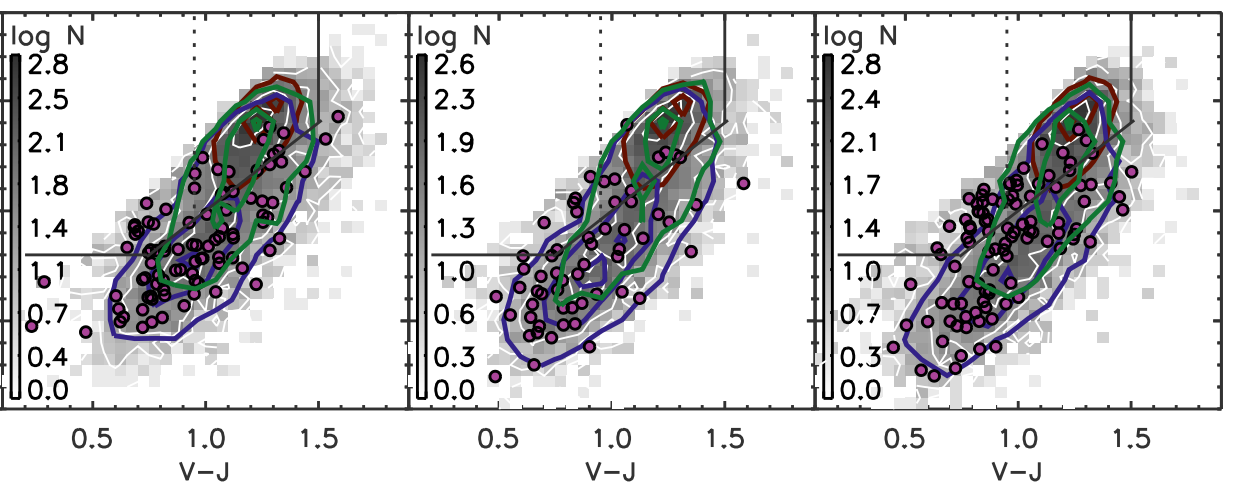

Figure B2. Rest-frame broad-band colours of spectrally classified galaxies in the GAMA survey in four redshift bins. The grey scale indicates the logarithmic number of objects in the parent sample with $\mathrm{S} / \mathrm{N}>6.5$ per $6 \AA$ pixel. Coloured contours show the different spectral classes in each panel, from left to right these are: PSB, quiescent, star-forming and green-valley galaxies. Contours show 10, 50 and 90 per cent of the maximum number of galaxies in each sample. Solid black lines are the commonly used separator between red-sequence, blue-sequence and dusty star-forming galaxies. The dotted black line from Whitaker et al. (2012) separates PSB galaxies from red-sequence galaxies.

we would see a shift in the locus of each spectral class from the broad-band classification in a systematic way. We do not see any shift in the locus of any spectral class with redshift, therefore we see no evidence that aperture bias causes us to misclassify large numbers of galaxies of any spectral class.

\section{APPENDIX C: COMPARISON TO LITERATURE MASS FUNCTIONS}

In Fig. C1, we compare our mass functions to those in the literature to check that our QSR completeness correction for spectra below our $\mathrm{S} / \mathrm{N}$ threshold allows us to correctly recover the mass functions. Although the Baldry et al. (2012) mass functions are

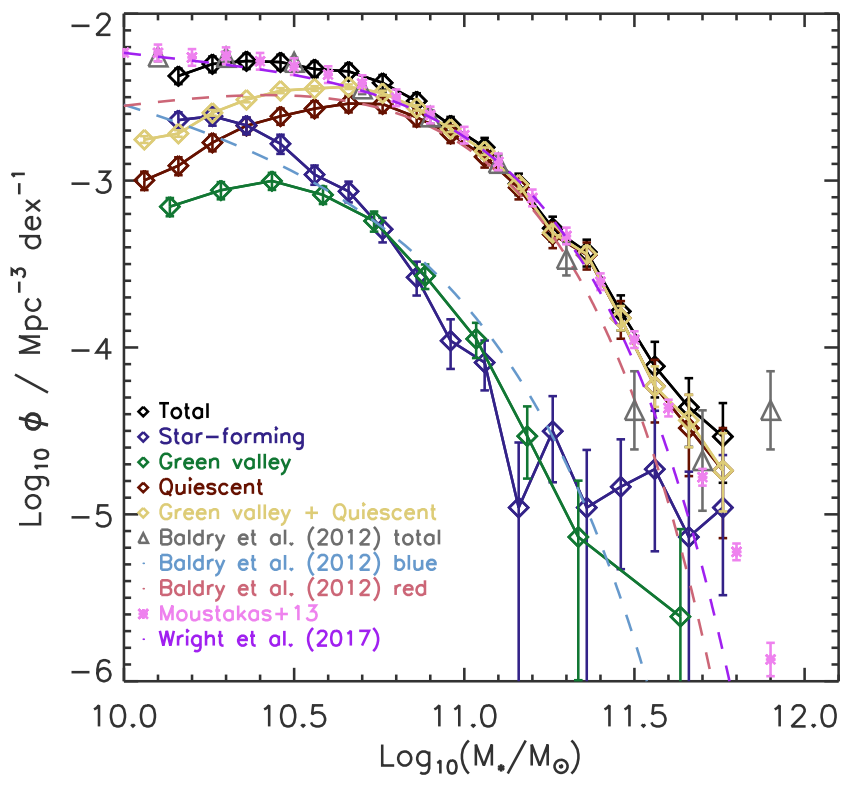

Figure C1. The completeness corrected mass functions of the total, quiescent population, star-forming populations, green valley, and combined quiescent population and green-valley populations for our lowest redshift bin $(0.05<z<0.14)$ compared to those in the literature. Errors on mass functions from our study include Poisson, cosmic variance contributions and uncertainties propagated from the uncertainty on fitting the stellar masses. derived from GAMA galaxies at $0<z<0.06$ and our sample spans $0.05<z<0.14$, there should be negligible evolution in this small redshift range. Our total and star-forming mass functions trace the Baldry et al. (2012) mass functions well, which shows that our completeness corrections are correct. Furthermore, we find excellent agreement between our total mass function and that from Moustakas et al. (2013) and Wright et al. (2017).

There is a slight deficit of low-mass quiescent galaxies in our sample compared to Baldry et al. (2012). This may be due to the differences in sample selection, as Baldry et al. (2012) separate starforming and quiescent galaxies using the $u-r$ optical colour, and we use a cleaner spectroscopic selection that likely has less contamination by dusty objects. Furthermore, we separate quiescent from green-valley galaxies, whereas Baldry et al. (2012) do not make this discrimination, meaning that green-valley galaxies will be mixed with the red and blue populations defined on broad-band colours. Adding the quiescent population and green-valley mass functions together, they approximately reproduce the Baldry et al. (2012) quiescent population mass function, with a slight deficit remaining as a small number of galaxies which are red in $u-r$ are classified spectroscopically as star forming. The deficit of spectroscopic quiescent galaxies at the low-mass end compared to Baldry et al. (2012) suggests that broad-band optical colour selection tends to classify spectroscopic green-valley galaxies as red. Additionally, Baldry et al. (2012) calculated stellar masses with ugriz data only, whereas we derive more robust stellar mass measurements by using UV and NIR data in addition to ugriz data, which may account for small differences in the mass functions. Note that Wright et al. (2017) use updated GAMA photometry and we find excellent agreement with their mass function.

In Fig. C2, we compare our VIPERS mass functions to those in Davidzon et al. (2013), Ilbert et al. (2013), Moustakas et al. (2013), Muzzin et al. (2013) and Tomczak et al. (2014) using similar redshift ranges and cosmology. In all redshift bins, we see good agreement with most literature studies. Our mass function has slightly different number densities in the $0.65<z<0.8$ bin, which may be caused by mismatches in the redshift ranges of literature studies, by different stellar population models and/or fitting methods, or dust laws used by each study. Note that we use the same redshift ranges as Moustakas et al. (2013) and we see an excellent agreement with this study in all redshift bins. There is no trend of worsening discrepancy with 


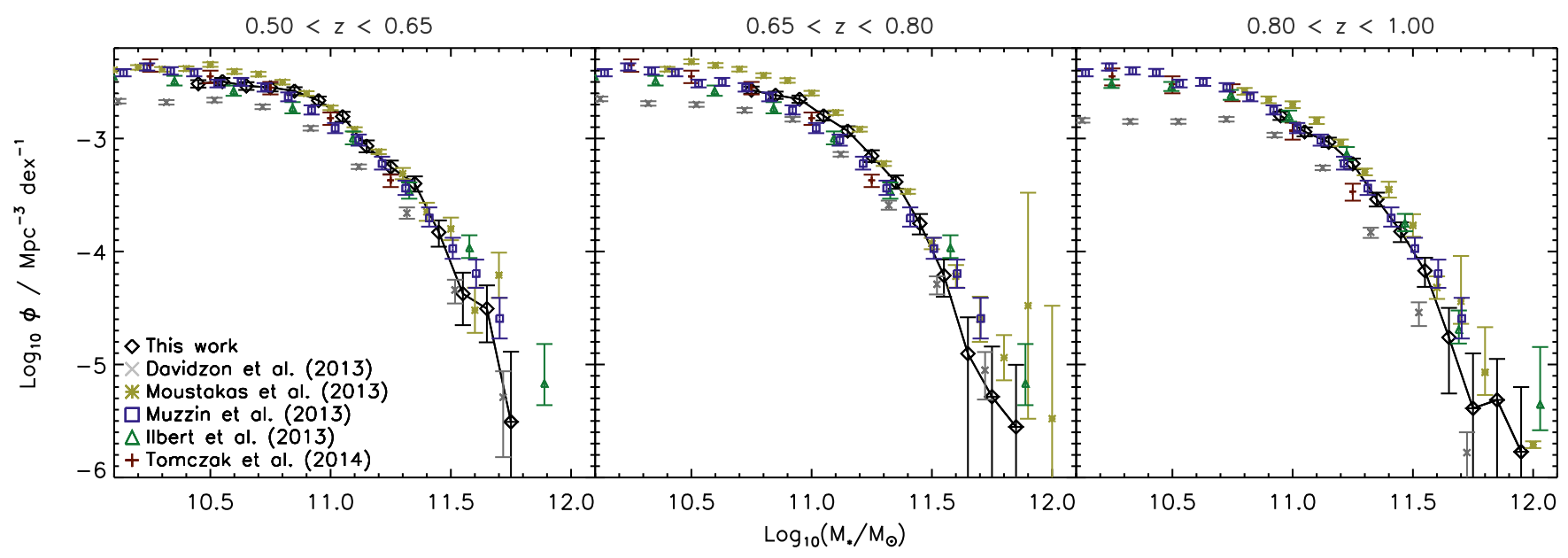

Figure C2. The completeness corrected total mass functions in this work (black diamonds) compared to those in Davidzon et al. (2013), Ilbert et al. (2013), Moustakas et al. (2013), Muzzin et al. (2013) and Tomczak et al. (2014). All points have been scaled to the same cosmology. Errors on the black points include Poisson, cosmic variance contributions and uncertainties propagated from the uncertainty on fitting the stellar masses.

most literature mass functions with increasing redshift. However, after scaling our masses to the same cosmology, we find that the mass functions from Davidzon et al. (2013) show an increasing discrepancy with redshift compared to our total mass functions. Whilst we do not use exactly the same redshift bins or stellar population models, the strong redshift trend suggests that this is unlikely to be the source of the difference between our mass functions. As the stellar masses from Davidzon et al. (2013) are not publicly available we are unable to dig deeper into this discrepancy.

${ }^{1}$ School of Physics and Astronomy (SUPA), University of St Andrews, North Haugh, St Andrews, Fife, KY16 9SS, UK

${ }^{2}$ Department of Physics and Astronomy, Johns Hopkins University, Bloomberg Center, 3400 N. Charles St., Baltimore, MD 21218, USA

${ }^{3}$ Institute for Astronomy, University of Edinburgh, Royal Observatory, Blackford Hill, Edinburgh EH9 3HJ, UK

${ }^{4}$ Astrophysics Group, School of Physics, University of Bristol, Tyndall Avenue, Bristol BS8 1TL, UK

${ }^{5}$ Australian Astronomical Observatory, PO Box 915, North Ryde, NSW 1670, Australia

${ }^{6}$ ICRAR, The University of Western Australia, 35 Stirling Highway, Crawley, WA 6009, Australia

${ }^{7}$ Department of Physics and Astronomy, Macquarie University, NSW 2109, Australia
${ }^{8}$ E. A. Milne Centre for Astrophysics, University of Hull, Cottingham Road, Kingston-upon-Hull, HU6 7RX, UK

${ }^{9}$ School of Physics and Astronomy, Monash University, Clayton, Victoria 3800, Australia

${ }^{10}$ Jeremiah Horrocks Institute, University of Central Lancashire, PR1 2HE

Preston, UK

${ }^{11}$ SRON Netherlands Institute for Space Research, Landleven 12, NL-9747 $A D$ Groningen, the Netherlands

${ }^{12}$ Kapteyn Astronomical Institute, University of Groningen, Postbus 800, NL-9700 AV Groningen, the Netherlands

${ }^{13}$ NASA Ames Research Center, N232, Moffett Field, Mountain View, CA 94035, USA

${ }^{14}$ Sydney Institute for Astronomy, School of Physics A28, University of Sydney, NSW 2006, Australia

${ }^{15}$ Research School of Astronomy and Astrophysics, Australian National University, Canberra, ACT 2611, Australia

${ }^{16}$ University of Leiden, Sterrenwacht Leiden, Niels Bohrweg 2, NL-2333 CA Leiden, the Netherlands

${ }^{17}$ Department of Physics and Astronomy 102 Natural Science Building, University of Louisville, Louisville KY 40292, USA

${ }^{18}$ Centre for Astrophysics and Supercomputing, Swinburne University of Technology, Hawthorn 3122, Australia

This paper has been typeset from a $\mathrm{T}_{\mathrm{E}} \mathrm{X} / \mathrm{L} \mathrm{T}_{\mathrm{E}} \mathrm{X}$ file prepared by the author. 